\title{
Cylindrical RF Network Antennas for Coupled Plasma Sources Copper Legs Delayed in Time System Stability Analysis
}

\author{
Ofer Aluf \\ Physical Electronics Dept., Tel-Aviv University, Ramat-Aviv, Israel. \\ oferaluf@bezeqint.net
}

\begin{abstract}
In this article, Very Crucial subject discussed cylindrical (closed) RF network antennas for coupled plasma sources copper legs delayed in time system stability analysis. Resonant RF network antennas are important to plasma sources with many applications. The cylindrical resonant RF network antennas run as large volume plasma sources and have stability switching due to system's copper legs parasitic effects. The cylindrical RF network antenna structure is 16-leg cylindrical (Birdcage) RF antenna which has electrical circuit and opposite points of RF feeding and grounding. The vacuum vessel is a glass cylinder closed at the top and bottom by grounding metal plates. Generally there are two popular different resonant RF network assemblies: a cylindrical and a planar RF antenna. The cylindrical RF antenna is built as a high-pass Birdcage coil. The antenna is mounted outside a glass tube. The RF antenna consists of 16 copper legs equally spaced interconnected with capacitors. Due to RF antenna copper leg parasitic effect we get copper leg's current and current derivative with delay $\tau_{1-k}$ and $\tau_{2-k}$ ( $k$ is leg number index, $k=1, \ldots, 16)$. The uncooled antenna is fed at the midpoint and operated with opposite grounded. Alternatively, it can be fed by another transmitter unit. Due to cylindrical antenna parasitic delayed in time, there is a stability issue by analyzing its operation. We consider for simplicity that all copper leg's current parasitic time delayed are equal $\left(\tau_{1-1}=\tau_{1-2}=\ldots=\tau_{1-16}\right)$ and current derivative parasitic time delayed are equal $\left(\tau_{2-1}=\tau_{2-2}=\ldots=\tau_{2-16}\right)$. The cylindrical RF network antennas delayed in time equivalent circuit can represent as a delayed differential equations which depend on variable parameters and delays. The investigation of our cylindrical network antenna with copper leg system, a differential equation is based on bifurcation theory [1], a study of possible changes in the structure of the orbits of a delayed differential equation depending on variable parameters. Cylindrical RF network antenna analysis is done under two series of different time delays respect to antenna's copper legs current and current derivative. All of that for optimization of a cylindrical RF network antenna circuit parameter analysis to get the best performance. The cylindrical network antenna with copper leg system can be represented as delayed differential equations which, depending on variable parameters and delays. There is a practical guideline that combines graphical information with analytical work to effectively study the local stability of models involving delay dependent parameters. The stability of a given steady state is determined by the graphs of some function of $\tau_{\mathrm{i}-1}, . ., \tau_{\mathrm{i}-16}(\mathrm{i}=1,2)$ [2] [3] [4].

Index Terms - Cylindrical RF network antenna, Delay Differential Equations (DDE), Stability, Bifurcation, Orbit.
\end{abstract}




\section{Introduction}

In this article, Very Critical and useful subject is discussed: cylindrical (closed) RF network antennas for coupled plasma sources copper legs delayed in time. The resonant RF networks can be arranged to form large-area or large-volume plasma sources with properties similar to Inductive Coupled Plasma (ICP) devices. There are medical applications of Birdcage coils and closed and open configurations of the antenna for plasma production are possible and can be analyzed by using mathematical formulation. There are systems of an open network antenna as a large-area planar plasma source and of a closed network antenna as a cylindrical plasma source. Both are composed of similar electrical meshes. Operation at different normal modes shows the capability of this antenna type of large-volume plasma applications.

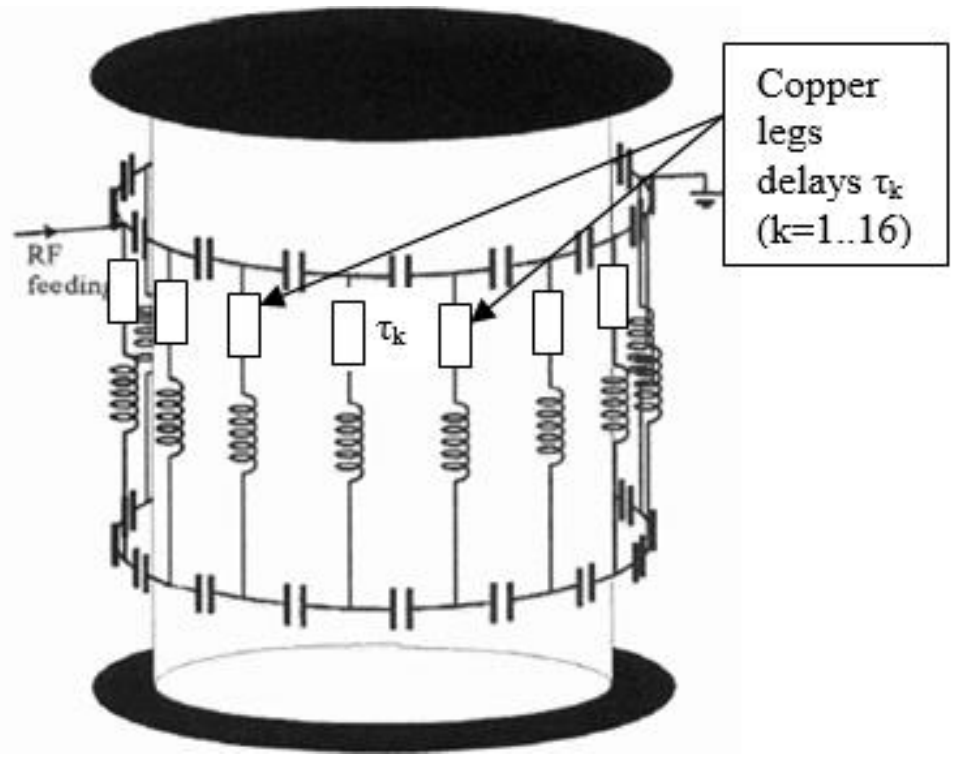

Figure 1. Schematic of the 16-leg cylindrical (Birdcage) RF Network antenna (closed).

An important issue of proper antenna operation is the location of the RF feeding and grounding connections on the antenna. There are a large number of different RF antenna arrangements possible in view of the geometry and RF operation and of plasma obtained. In this paper, we investigated only cylindrical RF antenna which built following a high-pass Birdcage coil. The antenna is mounted outside a glass tube. The RF antenna consists of 16 copper legs (Fig. 1), equally spaced interconnected with capacitors, each copper leg current has parasitic time delayed $\left(\tau_{1-1 \ldots} \tau_{1-16}\right)$. We consider for simplicity that all copper legs parasitic time delayed are equal $\left(\tau_{1-1}=\tau_{1-2}=\ldots=\tau_{1-16}\right)$ and the voltages on delay units $\left(V_{\varepsilon}\right)$ are neglected $V_{\varepsilon} \rightarrow \varepsilon$. There is a delay in each Copper leg current $I_{1}\left(t-\tau_{1-1}\right), \ldots, I_{16}\left(t-\tau_{1-16}\right)$. We consider all interconnected capacitor values are the same (C) and all antenna elements inductance values are the same $(L)$.

$$
\begin{aligned}
& C_{A 1}=C_{A 2}=\ldots=C_{A 16}=C ; C_{B 1}=C_{B 2}=\ldots=C_{B 16}=C \\
& L_{1}=L_{2}=\ldots=L_{16}=L ; I_{L 1}=I_{1}, I_{L 2}=I_{2}, \ldots, I_{L 16}=I_{16}
\end{aligned}
$$

We choose first case: antenna network is fed by the transmitter unit (S1=OFF, no direct RF feeding). The upper view of 16-leg cylindrical RF antenna network described in Fig. 2. 


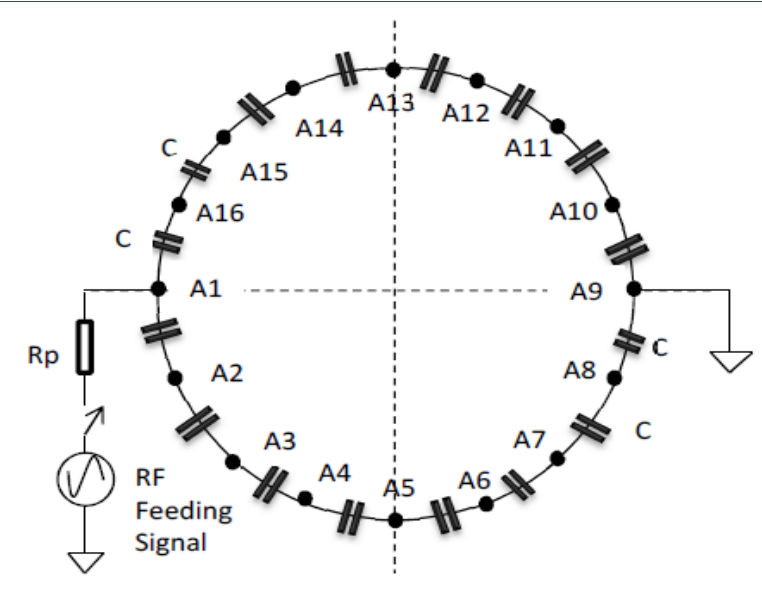

Figure 2. Upper view of 16-leg cylindrical RF antenna.

The lower view of 16-leg cylindrical RF antenna network described in Fig. 3.

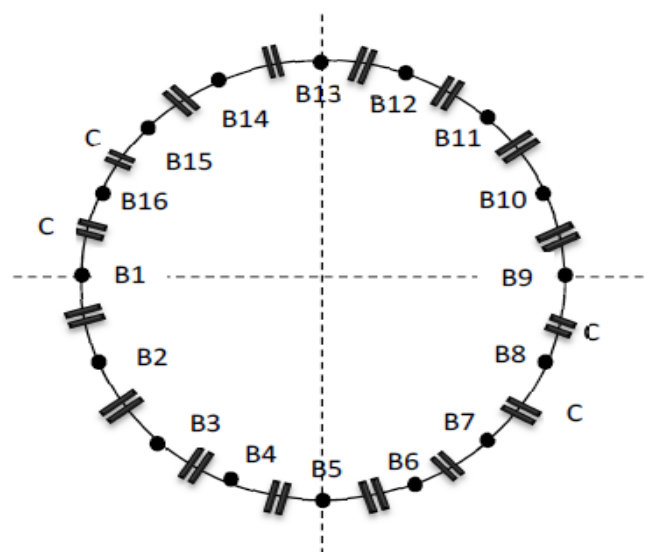

Figure 3. Lower view of 16-leg cylindrical RF antenna

\section{Cylindrical RF Network Antennas Equivalent Circuit and represent Delay Differential Equations}

Cylindrical RF network antenna system can represent as round strip of capacitors and inductors (Fig. 4a \& 4b). The schematic contains RF feeding signal, S1 switch ( $\mathrm{S} 1=\mathrm{ON}$ for direct RF signal feeding, S1=OFF for RF signal transmitter feeding). The upper network connecting nodes are A1, A2,..,A16 and the lower network connecting nodes are B1, B2,..,B16. Antenna copper leg current parasitic delays are represented by delay units $\operatorname{Tau}_{1-1 \ldots} \operatorname{Tau}_{1-16}\left(\tau_{1-1}, . ., \tau_{1-16}\right)$. Rp is the parasitic resistance of RF feeding point (A1). The upper system spaced capacitors are CA1,..,CA16 and the lower system spaced capacitors are $\mathrm{CB} 1, \ldots, \mathrm{CB} 16$ [1] [2]. 


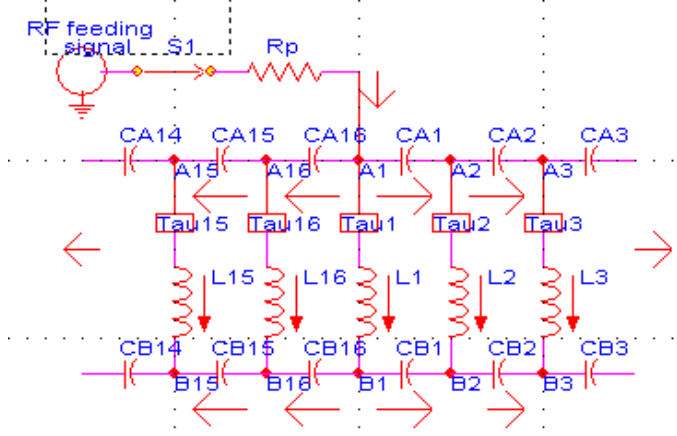

Figure 4a. 16-leg cylindrical RF antenna strip (feeding side)

\begin{tabular}{|l|l|l|l|}
\hline$I_{C A 1}=C_{A 1} \cdot \frac{d}{d t}\left(V_{A 1}-V_{A 2}\right), I_{C A 2}=C_{A 2} \cdot \frac{d}{d t}\left(V_{A 2}-V_{A 3}\right)$ & 3 & $I_{C A 3}=C_{A 3} \cdot \frac{d}{d t}\left(V_{A 3}-V_{A 4}\right), \ldots, I_{C A 7}=C_{A 7} \cdot \frac{d}{d t}\left(V_{A 7}-V_{A 8}\right)$ & 4 \\
\hline$I_{C A 8}=C_{A 8} \cdot \frac{d V_{A 8}}{d t} ; I_{C A k}=C_{A k} \cdot \frac{d}{d t}\left(V_{A k}-V_{A(k+1)}\right) ; k=1, \ldots, 7$ & 5 & $\begin{array}{l}I_{C A 16}=C_{A 16} \cdot \frac{d}{d t}\left(V_{A 1}-V_{A 16}\right), I_{C A 15}=C_{A 15} \cdot \frac{d}{d t}\left(V_{A 16}-V_{A 15}\right) \\
I_{C A 14}=C_{A 14} \cdot \frac{d}{d t}\left(V_{A 15}-V_{A 14}\right), \ldots, I_{C A 10}=C_{A 10} \cdot \frac{d}{d t}\left(V_{A 11}-V_{A 10}\right)\end{array}$ & 6 \\
\hline$I_{C A 9}=C_{A 9} \cdot \frac{d V_{A 10}}{d t} ; I_{C A l}=C_{A l} \cdot \frac{d}{d t}\left(V_{A(l+1)}-V_{A l}\right) ; l=10, \ldots, 15$ & 7 & & \\
\hline
\end{tabular}

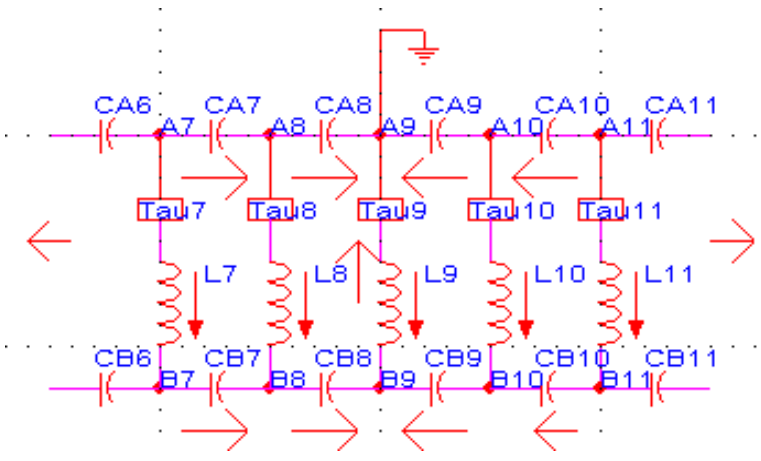

Figure 4b. 16-leg cylindrical RF antenna strip (ground side)

\begin{tabular}{|c|c|c|c|}
\hline $\begin{array}{l}I_{C B 1}=C_{B 1} \cdot \frac{d}{d t}\left(V_{B 1}-V_{B 2}\right), I_{C B 2}=C_{B 2} \cdot \frac{d}{d t}\left(V_{B 2}-V_{B 3}\right) ; k=1, \ldots, 8 \\
, \ldots, I_{C B 8}=C_{B 8} \cdot \frac{d}{d t}\left(V_{B 2}-V_{B 3}\right) ; I_{C B k}=C_{B k} \cdot \frac{d}{d t}\left(V_{B k}-V_{B(k+1)}\right)\end{array}$ & 8 & $\begin{array}{l}I_{C B 16}=C_{B 16} \cdot \frac{d}{d t}\left(V_{B 1}-V_{B 16}\right), I_{C B 15}=C_{B 15} \cdot \frac{d}{d t}\left(V_{B 16}-V_{B 15}\right) \\
, \ldots, I_{C B 9}=C_{B 9} \cdot \frac{d}{d t}\left(V_{B 10}-V_{B 9}\right) ; I_{C B l}=C_{B l} \cdot \frac{d}{d t}\left(V_{B(l+1)}-V_{B l}\right) \\
l=15, \ldots, 9\end{array}$ & 9 \\
\hline $\begin{array}{l}V_{A 1}-V_{B 1}=L_{1} \cdot \frac{d I_{L 1}}{d t} ; V_{A 2}-V_{B 2}=L_{2} \cdot \frac{d I_{L 2}}{d t} ; V_{A 3}-V_{B 3}=L_{3} \cdot \frac{d I_{L 3}}{d t} \\
, \ldots, V_{A 8}-V_{B 8}=L_{8} \cdot \frac{d I_{L 8}}{d t} ; V_{B 9}=L_{9} \cdot \frac{d I_{L 9}}{d t} ; V_{A 10}-V_{B 10}=L_{10} \cdot \frac{d I_{L 10}}{d t} \\
, \ldots, V_{A 16}-V_{B 16}=L_{16} \cdot \frac{d I_{L 16}}{d t} ; V_{A m}-V_{B m}=L_{m} \cdot \frac{d I_{L m}}{d t} ; m=1, \ldots, 16 ; m \neq 9\end{array}$ & 10 & $V_{B 9}=L_{9} \cdot \frac{d I_{L 9}}{d t} ; V_{A 9}=0 ; A 9-$ ground & 11 \\
\hline $\begin{array}{l}I_{R_{P}}=I_{C A 16}+I_{C A 1}+I_{L 1} ; I_{C A 1}=I_{C A 2}+I_{L 2} ; I_{C A 2}=I_{C A 3}+I_{L 3} \\
, \ldots, I_{C A 7}=I_{C A 8}+I_{L 8} ; I_{C A l}=I_{C A(l+1)}+I_{L(l+1)} ; l=1, \ldots, 7\end{array}$ & 12 & $\begin{array}{l}I_{C A 16}=I_{C A 15}+I_{L 16} ; I_{C A 15}=I_{C A 14}+I_{L 15} ; I_{C A 14}=I_{C A 13}+I_{L 14} \\
, \ldots, I_{C A 10}=I_{C A 9}+I_{L 10} ; I_{C A k}=I_{C A(k-1)}+I_{L k} ; k=16, \ldots, 10\end{array}$ & 13 \\
\hline$I_{L 1}=I_{C B 1}+I_{C B 16} ; I_{C B 2}=I_{C B 1}+I_{L 2} ; I_{C B 3}=I_{C B 2}+I_{L 3}$ & 14 & $I_{C B 4}=I_{C B 3}+I_{L 4}, \ldots, I_{C B 8}=I_{C B 7}+I_{L 8} ; I_{L 9}=I_{C B 8}+I_{C B 9}$ & 15 \\
\hline$I_{C B m}=I_{C B(m-1)}+I_{L m} ; m=2, \ldots, 8$ & 16 & $I_{C B 15}=I_{C B 16}+I_{L 16} ; I_{C B 14}=I_{C B 15}+I_{L 15} ; I_{C B 13}=I_{C B 14}+I_{L 14}$ & 17 \\
\hline$I_{C B 12}=I_{C B 13}+I_{L 13}, \ldots, I_{C B 9}=I_{C B 10}+I_{L 10}$ & 18 & $I_{C B n}=I_{C B(n+1)}+I_{L(n+1)} ; n=15, \ldots, 9$ & 19 \\
\hline
\end{tabular}




\section{Upon mathematic manipulation we get the following expressions:}

\begin{tabular}{|c|c|c|c|}
\hline$\frac{I_{C_{A 1}}}{C_{A 1}}-\frac{I_{C_{B 1}}}{C_{B 1}}=L_{1} \cdot \frac{d^{2} I_{L 1}}{d t^{2}}-L_{2} \cdot \frac{d^{2} I_{L 2}}{d t^{2}} ; C_{A 1}=C_{B 1}=C$ & 20 & $L_{1}=L_{2}=L ; \frac{1}{L C} \cdot\left(I_{C_{A 1}}-I_{C_{B 1}}\right)=\frac{d^{2} I_{L 1}}{d t^{2}}-\frac{d^{2} I_{L 2}}{d t^{2}}$ & 21 \\
\hline$\frac{I_{C_{A 2}}}{C_{A 2}}-\frac{I_{C_{B 2}}}{C_{B 2}}=L_{2} \cdot \frac{d^{2} I_{L 2}}{d t^{2}}-L_{3} \cdot \frac{d^{2} I_{L 3}}{d t^{2}} ; C_{A 2}=C_{B 2}=C$ & 22 & $L_{2}=L_{3}=L ; \frac{1}{L C} \cdot\left(I_{C_{A 2}}-I_{C_{B 2}}\right)=\frac{d^{2} I_{L 2}}{d t^{2}}-\frac{d^{2} I_{L 3}}{d t^{2}}$ & 23 \\
\hline$\ldots \ldots \frac{I_{C_{A 7}}}{C_{A 7}}-\frac{I_{C_{B 7}}}{C_{B 7}}=L_{7} \cdot \frac{d^{2} I_{L 7}}{d t^{2}}-L_{8} \cdot \frac{d^{2} I_{L 8}}{d t^{2}}$ & 24 & $L_{7}=L_{8}=L ; \frac{1}{L C} \cdot\left(I_{C_{A 7}}-I_{C_{B 7}}\right)=\frac{d^{2} I_{L 7}}{d t^{2}}-\frac{d^{2} I_{L 8}}{d t^{2}}$ & 25 \\
\hline$C_{A 7}=C_{B 7}=C ; L_{1}=L_{2}=\ldots=L_{16}=L ; k=1, \ldots, 7$ & 26 & $\frac{1}{L C} \cdot\left(I_{C_{A k}}-I_{C_{B k}}\right)=\frac{d^{2} I_{L k}}{d t^{2}}-\frac{d^{2} I_{L(k+1)}}{d t^{2}} ; k=1, \ldots, 7$ & 27 \\
\hline$\frac{1}{L C} \cdot\left(I_{C_{A 10}}-I_{C_{B 10}}\right)=\frac{d^{2} I_{L 11}}{d t^{2}}-\frac{d^{2} I_{L 10}}{d t^{2}} ; C_{A 10}=C_{B 10}=C$ & 28 & $\cdots \cdot \frac{1}{L C} \cdot\left(I_{C_{A 15}}-I_{C_{B 15}}\right)=\frac{d^{2} I_{L 16}}{d t^{2}}-\frac{d^{2} I_{L 15}}{d t^{2}} ; C_{A 15}=C_{B 15}=C$ & 29 \\
\hline$\frac{1}{L C} \cdot\left(I_{C_{A m}}-I_{C_{B m}}\right)=\frac{d^{2} I_{L(m+1)}}{d t^{2}}-\frac{d^{2} I_{L m}}{d t^{2}} ; m=10, \ldots, 15$ & 30 & $C_{A 8}=C_{B 8}=C ; V_{A 9}=0 ; \frac{1}{L C} \cdot\left(I_{C_{A 8}}-I_{C_{B 8}}\right)=\frac{d^{2} I_{L 8}}{d t^{2}}+\frac{d^{2} I_{L 9}}{d t^{2}}$ & 31 \\
\hline$C_{A 9}=C_{B 9}=C ; V_{A 9}=0 ; \frac{1}{L C} \cdot\left(I_{C_{A 9}}-I_{C_{B 9}}\right)=\frac{d^{2} I_{L 9}}{d t^{2}}+\frac{d^{2} I_{L 10}}{d t^{2}}$ & 32 & $C_{A 16}=C_{B 16}=C ; V_{A 9}=0 ; \frac{1}{L C} \cdot\left(I_{C_{A 16}}-I_{C_{B 16}}\right)=\frac{d^{2} I_{L 1}}{d t^{2}}-\frac{d^{2} I_{L 16}}{d t^{2}}$ & 33 \\
\hline$I_{R_{P}}=I_{C_{A 16}}+I_{C_{A 1}}+I_{L 1} ; I_{C_{A 1}}=I_{C_{A 2}}+I_{L 2} ; I_{C_{A 2}}=I_{C_{A 3}}+I_{L 3}$ & 34 & $I_{C_{A 3}}=I_{C_{A 4}}+I_{L 4} ; I_{C_{A 4}}=I_{C_{A 5}}+I_{L 5} ; I_{C_{A 5}}=I_{C_{A 6}}+I_{L 6}$ & 35 \\
\hline$I_{C_{A 6}}=I_{C_{A 7}}+I_{L 7} ; I_{C_{A 7}}=I_{C_{A 8}}+I_{L 8} ; I_{C_{A 10}}=I_{C_{A 9}}+I_{L 10}$ & 36 & $\begin{array}{c}I_{C_{A 11}}=I_{C_{A 10}}+I_{L 11} ; I_{C_{A 12}}=I_{C_{A 11}}+I_{L 12} ; I_{C_{A 13}}=I_{C_{A 12}}+I_{L 13} \\
I_{C_{A 14}}=I_{C_{A 13}}+I_{L 14} ; I_{C_{A 15}}=I_{C_{A 14}}+I_{L 15} ; I_{C_{A 16}}=I_{C_{A 15}}+I_{L 16} \\
I_{L 1}=I_{C_{B 1}}+I_{C_{B 16}} ; I_{L 9}=I_{C_{B 8}}+I_{C_{B 9}} ; I_{C_{B 2}}=I_{C_{B 1}}+I_{L 2}\end{array}$ & 37 \\
\hline$I_{C_{B 3}}=I_{C_{B 2}}+I_{L 3} ; I_{C_{B 4}}=I_{C_{B 3}}+I_{L 4} ; I_{C_{B 5}}=I_{C_{B 4}}+I_{L 5}$ & 38 & $I_{C_{B 6}}=I_{C_{B 5}}+I_{L 6} ; I_{C_{B 7}}=I_{C_{B 6}}+I_{L 7} ; I_{C_{B 8}}=I_{C_{B 7}}+I_{L 8}$ & 39 \\
\hline$I_{C_{B 9}}=I_{C_{B 10}}+I_{L 10} ; I_{C_{B 10}}=I_{C_{B 11}}+I_{L 11} ; I_{C_{B 11}}=I_{C_{B 12}}+I_{L 12}$ & 40 & $\begin{array}{l}I_{C_{B 12}}=I_{C_{B 13}}+I_{L 13} ; I_{C_{B 13}}=I_{C_{B 14}}+I_{L 14} ; I_{C_{B 14}}=I_{C_{B 15}}+I_{L 15} \\
I_{C_{B 15}}=I_{C_{B 16}}+I_{L 16}\end{array}$ & 41 \\
\hline
\end{tabular}

S1 is OFF for RF signal transmitter feeding.

\begin{tabular}{|c|c|c|c|}
\hline$I_{R_{P}}=0 \Rightarrow I_{C_{A 16}}+I_{C_{A 1}}+I_{L 1}=0$ & 42 & $I_{C_{A 1}}=I_{C_{A B}}+\sum_{k=2}^{8} I_{L k} ; I_{C_{A 2}}=I_{C_{A B}}+\sum_{k=3}^{8} I_{L k} ; I_{C_{A 3}}=I_{C_{A B}}+\sum_{k=4}^{8} I_{L k}$ & 43 \\
\hline$I_{C_{A 4}}=I_{C_{A B}}+\sum_{k=5}^{8} I_{L k} ; I_{C_{A 5}}=I_{C_{A 8}}+\sum_{k=6}^{8} I_{L k} ; I_{C_{A 6}}=I_{C_{A B}}+\sum_{k=7}^{8} I_{L k}$ & 44 & $I_{C_{A 7}}=I_{C_{A 8}}+I_{L 8} ; I_{C_{A 16}}=I_{C_{A 9}}+\sum_{k=10}^{16} I_{L k} ; I_{C_{A 15}}=I_{C_{A 9}}+\sum_{k=10}^{15} I_{L k}$ & 45 \\
\hline$I_{C_{A 14}}=I_{C_{A 9}}+\sum_{k=10}^{14} I_{L k} ; I_{C_{A 13}}=I_{C_{A 9}}+\sum_{k=10}^{13} I_{L k} ; I_{C_{A 12}}=I_{C_{A 9}}+\sum_{k=10}^{12} I_{L k}$ & 46 & $I_{C_{A 11}}=I_{C_{A 9}}+\sum_{k=10}^{11} I_{L k} ; I_{C_{A 10}}=I_{C_{A 9}}+I_{L_{10}}$ & 47 \\
\hline$I_{C_{B 1}}=I_{L 9}-I_{C_{B 16}}-\sum_{k=2, k \neq 9}^{16} I_{L k} ; I_{C_{B 2}}=I_{L 9}-I_{C_{B 16}}-\sum_{k=3, k \neq 9}^{16} I_{L k}$ & 48 & $I_{C_{B 3}}=I_{L 9}-I_{C_{B 16}}-\sum_{k=4, k \neq 9}^{16} I_{L k} ; I_{C_{B 4}}=I_{L 9}-I_{C_{B 16}}-\sum_{k=5, k \neq 9}^{16} I_{L k}$ & 49 \\
\hline$I_{C_{B 5}}=I_{L 9}-I_{C_{B 16}}-\sum_{k=6, k \neq 9}^{16} I_{L k} ; I_{C_{B 6}}=I_{L 9}-I_{C_{B 16}}-\sum_{k=7, k \neq 9}^{16} I_{L k}$ & 50 & $I_{C_{B 7}}=I_{L 9}-I_{C_{B 16}}-\sum_{k=8, k \neq 9}^{16} I_{L k} ; I_{C_{B 8}}=I_{L 9}-I_{C_{B 16}}-\sum_{k=10}^{16} I_{L k}$ & 51 \\
\hline$I_{C_{B 9}}=I_{C_{B 16}}+\sum_{k=10}^{16} I_{L k} ; I_{C_{B 10}}=I_{C_{B 16}}+\sum_{k=11}^{16} I_{L k}$ & 52 & $I_{C_{B 11}}=I_{C_{B 16}}+\sum_{k=12}^{16} I_{L k} ; I_{C_{B 12}}=I_{C_{B 16}}+\sum_{k=13}^{16} I_{L k}$ & 53 \\
\hline$I_{C_{B 13}}=I_{C_{B 16}}+\sum_{k=14}^{16} I_{L k} ; I_{C_{B 14}}=I_{C_{B 16}}+\sum_{k=15}^{16} I_{L k}$ & 54 & $I_{C_{B 15}}=I_{C_{B 16}}+I_{L 16} ; I_{L 1}=I_{C_{B 1}}+I_{C_{B 16}}$ & 55 \\
\hline
\end{tabular}

\section{We get the following additional exppressions:}




\begin{tabular}{|l|l|l|l|l|}
\hline$\frac{2}{L \cdot C} \cdot\left(I_{L 2}-I_{L 4}\right)=\frac{d^{2} I_{L 1}}{d t^{2}}-\frac{d^{2} I_{L 5}}{d t^{2}}-2 \cdot\left[\frac{d^{2} I_{L 2}}{d t^{2}}-\frac{d^{2} I_{L 4}}{d t^{2}}\right]$ & 56 & $\frac{2}{L \cdot C} \cdot\left(I_{L 6}-I_{L 8}\right)=\frac{d^{2} I_{L 5}}{d t^{2}}+\frac{d^{2} I_{L 9}}{d t^{2}}-2 \cdot\left[\frac{d^{2} I_{L 6}}{d t^{2}}-\frac{d^{2} I_{L 8}}{d t^{2}}\right]$ & 57 \\
\hline$\frac{-2}{L \cdot C} \cdot\left(I_{L 10}-I_{L 12}\right)=\frac{d^{2} I_{L 9}}{d t^{2}}+\frac{d^{2} I_{L 13}}{d t^{2}}+2 \cdot\left[\frac{d^{2} I_{L 10}}{d t^{2}}-\frac{d^{2} I_{L 12}}{d t^{2}}\right]$ & 58 & $\frac{-2}{L \cdot C} \cdot\left(I_{L 14}-I_{L 16}\right)=\frac{d^{2} I_{L 1}}{d t^{2}}-\frac{d^{2} I_{L 13}}{d t^{2}}+2 \cdot\left[\frac{d^{2} I_{L 14}}{d t^{2}}-\frac{d^{2} I_{L 16}}{d t^{2}}\right]$ & 60 \\
\hline
\end{tabular}

We add the first and second above equations:

$$
\begin{aligned}
{\left[{ }^{*}\right] } & \frac{2}{L \cdot C} \cdot\left\{\left(I_{L 2}-I_{L 4}\right)+\left(I_{L 6}-I_{L 8}\right)\right\}=\frac{d^{2} I_{L 1}}{d t^{2}} \\
+ & \frac{d^{2} I_{L 9}}{d t^{2}}-2 \cdot\left[\frac{d^{2} I_{L 2}}{d t^{2}}-\frac{d^{2} I_{L 4}}{d t^{2}}+\frac{d^{2} I_{L 6}}{d t^{2}}-\frac{d^{2} I_{L 8}}{d t^{2}}\right]
\end{aligned}
$$

We add the third and forth above equations:

$$
\begin{aligned}
{[* *] } & \frac{-2}{L \cdot C} \cdot\left\{\left(I_{L 10}-I_{L 12}\right)+\left(I_{L 14}-I_{L 16}\right)\right\}=\frac{d^{2} I_{L 1}}{d t^{2}} \\
& +\frac{d^{2} I_{L 9}}{d t^{2}}+2 \cdot\left[\frac{d^{2} I_{L 10}}{d t^{2}}-\frac{d^{2} I_{L 12}}{d t^{2}}+\frac{d^{2} I_{L 14}}{d t^{2}}-\frac{d^{2} I_{L 16}}{d t^{2}}\right]
\end{aligned}
$$

Integrating the last two results $\left(\left[{ }^{* *}\right]-[*]\right)$ gives the following:

$$
\begin{aligned}
& \frac{-2}{L \cdot C} \cdot\left\{I_{L 10}-I_{L 12}+I_{L 14}-I_{L 16}+I_{L 2}-I_{L 4}+I_{L 6}-I_{L 8}\right\} \\
& =2 \cdot\left[\frac{d^{2} I_{L 10}}{d t^{2}}-\frac{d^{2} I_{L 12}}{d t^{2}}+\frac{d^{2} I_{L 14}}{d t^{2}}-\frac{d^{2} I_{L 16}}{d t^{2}}+\frac{d^{2} I_{L 2}}{d t^{2}}-\frac{d^{2} I_{L 4}}{d t^{2}}\right. \\
& \left.+\frac{d^{2} I_{L 6}}{d t^{2}}-\frac{d^{2} I_{L 8}}{d t^{2}}\right]
\end{aligned}
$$

We define new global variables for our Cylindrical RF network antennas system.

$$
\begin{gathered}
Y=I_{L 10}-I_{L 12}+I_{L 14}-I_{L 16}+I_{L 2}-I_{L 4}+I_{L 6}-I_{L 8} \\
X=\frac{d I_{L 10}}{d t}-\frac{d I_{L 12}}{d t}+\frac{d I_{L 14}}{d t}-\frac{d I_{L 16}}{d t}+\frac{d I_{L 2}}{d t}-\frac{d I_{L 4}}{d t} \\
+\frac{d I_{L 6}}{d t}-\frac{d I_{L 8}}{d t} ; \frac{d Y}{d t}=X ; \frac{d X}{d t}=\frac{-1}{L \cdot C} \cdot Y
\end{gathered}
$$

Due to RF antenna copper leg parasitic effect, we get copper leg's current and current derivative with delay $\tau_{1-k}$ and $\tau_{2-k}(k$ is leg number index, $k=1, \ldots, 16)$. We consider for simplicity

$\tau_{1-1}=\tau_{1-2}=\ldots=\tau_{1-16} ; \tau_{2-1}=\tau_{2-2}=\ldots=\tau_{2-16 .} I_{L k}(t) \rightarrow I_{L k}\left(t-\tau_{1-k}\right) I_{L k}^{\prime}(t)=\frac{d I_{L k}(t)}{d t} ; I_{L k}^{\prime}(t) \rightarrow I_{L k}^{\prime}\left(t-\tau_{2-k}\right)$.

We consider no delay effect on 


$$
\begin{gathered}
\frac{d I_{L k}^{\prime}(t)}{d t} . Y(t) \rightarrow Y\left(t-\tau_{1}\right) ; X(t) \rightarrow X\left(t-\tau_{2}\right) . \\
\tau_{1}=\tau_{1-1}=\tau_{1-2}=\ldots=\tau_{1-16} ; \tau_{2}=\tau_{2-1}=\tau_{2-2}=\ldots=\tau_{2-16 .} . \\
\frac{d Y}{d t}=X\left(t-\tau_{2}\right) ; \frac{d X}{d t}=\frac{-1}{L \cdot C} \cdot Y\left(t-\tau_{1}\right)
\end{gathered}
$$

To find the Equilibrium points (fixed points) of the Cylindrical RF network antennas system is by

$$
\begin{gathered}
\lim _{t \rightarrow \infty} Y\left(t-\tau_{1}\right)=Y(t) \text { and } \lim _{t \rightarrow \infty} X\left(t-\tau_{2}\right)=X(t) . \frac{d Y}{d t}=0 ; \frac{d X}{d t}=0 \\
\forall \mathrm{t} \gg \tau_{1} ; \mathrm{t} \gg \tau_{2} \exists\left(t-\tau_{1}\right) \approx t ;\left(t-\tau_{2}\right) \approx t, \mathrm{t} \rightarrow \infty
\end{gathered}
$$

We get two equations and the only fixed point is

$$
\begin{gathered}
E^{(0)}\left(Y^{(0)}, X^{(0)}\right)=(0,0) . \\
Y^{(0)}=I_{L 10}^{(0)}-I_{L 12}^{(0)}+I_{L 14}^{(0)}-I_{L 16}^{(0)}+I_{L 2}^{(0)}-I_{L 4}^{(0)}+I_{L 6}^{(0)}-I_{L 8}^{(0)}=0 \\
X^{(0)}=I_{L 10}^{(0)}-I_{L 12}^{\prime(0)}+I_{L 14}^{\prime(0)}-I_{L 16}^{(0)}+I_{L 2}^{(0)}-I_{L 4}^{(0)}+I_{L 6}^{\prime(0)}-I_{L 8}^{\prime(0)}=0
\end{gathered}
$$

Stability analysis : The standard local stability analysis about any one of the equilibrium points of Cylindrical RF network antennas system consists in adding to coordinates [ $\mathrm{Y} X]$ arbitrarily small increments of exponential form $[y x] \cdot e^{\lambda \cdot t}$, and retaining the first order terms in $\mathrm{y}, \mathrm{x}$. The system of two homogeneous equations leads to a polynomial characteristics equation in the Eigen values $\lambda$. The polynomial characteristics equations accept by set the below current and current derivative respect to time into two Cylindrical RF network antennas system equations. Cylindrical RF network antennas system fixed values with arbitrarily small increments of exponential form $[\mathrm{y} x] . e^{\lambda . t}$ are: $\mathrm{i}=0$ (first fixed point), $\mathrm{i}=1$ (second fixed point), $\mathrm{i}=2$ (third fixed point).

$$
\begin{aligned}
& Y(t)=Y^{(i)}+y \cdot e^{\lambda \cdot t} ; X=X^{(i)}+x \cdot e^{\lambda \cdot t} ; Y\left(t-\tau_{1}\right)=Y^{(i)}+y \cdot e^{\lambda \cdot\left(t-\tau_{1}\right)} \\
& X\left(t-\tau_{2}\right)=X^{(i)}+x \cdot e^{\lambda \cdot\left(t-\tau_{2}\right)} \forall i=0,1,2
\end{aligned}
$$

We choose the above expressions for our $Y(t), X(t)$ as small displacement [y $\mathrm{x}$ ] from the system fixed points at time $\mathrm{t}=0$.

$$
Y(t=0)=Y^{(i)}+y ; X(t=0)=X^{(i)}+x
$$

for $\lambda<0, \mathrm{t}>0$ the selected fixed point is stable otherwise $\lambda>0, \mathrm{t}>0$ is Unstable. Our Cylindrical RF network antennas system tend to the selected fixed point exponentially for $\lambda<0, \mathrm{t}>0$ otherwise go away from the selected fixed point exponentially. $\lambda$ is the eigenvalue parameter which establish if the fixed point is stable or unstable, additionally his absolute value $(|\lambda|)$ establish the speed of flow toward or away from the selected fixed point [1] [2]. 
Table-1. Cylindrical RF network antennas system eigenvalues options

\begin{tabular}{|l|c|c|}
\hline & $\boldsymbol{\lambda}<\mathbf{0}$ & $\boldsymbol{\lambda}>\mathbf{0}$ \\
\hline $\mathbf{t}=\mathbf{0}$ & $\mathrm{Y}(\mathrm{t}=0)=\mathrm{Y}^{(\mathrm{i})}+\mathrm{y}$ & $\mathrm{Y}(\mathrm{t}=0)=\mathrm{Y}^{(\mathrm{i})}+\mathrm{y}$ \\
& $\mathrm{X}(\mathrm{t}=0)=\mathrm{X}^{(\mathrm{i})}+\mathrm{x}$ & $\mathrm{X}(\mathrm{t}=0)=\mathrm{X}^{(\mathrm{i})}+\mathrm{x}$ \\
\hline $\mathbf{t}>\mathbf{0}$ & $\mathrm{Y}(\mathrm{t})=\mathrm{Y}^{(\mathrm{i})}+\mathrm{y} \cdot \mathrm{e}^{-\lambda \mid \bullet t}$ & $\mathrm{Y}(\mathrm{t})=\mathrm{Y}^{(\mathrm{i})}+\mathrm{y} \cdot \mathrm{e}^{|\lambda| \bullet t}$ \\
& $\mathrm{X}(\mathrm{t})=\mathrm{X}^{(\mathrm{i})}+\mathrm{x} \cdot \mathrm{e}^{-\lambda \mid \bullet t}$ & $\mathrm{X}(\mathrm{t})=\mathrm{X}^{(\mathrm{i})}+\mathrm{x} \cdot \mathrm{e}^{|\lambda| \bullet t}$ \\
\hline $\mathbf{t} \rightarrow \infty$ & $\mathrm{Y}(\mathrm{t} \rightarrow \infty)=\mathrm{Y}^{(\mathrm{i})}$ & $\mathrm{Y}(\mathrm{t} \rightarrow \infty, \lambda>0)^{\sim} \mathrm{ye}^{|\lambda| \bullet t}$ \\
& $\mathrm{X}(\mathrm{t} \rightarrow \infty)=\mathrm{X}^{(\mathrm{i})}$ & $\mathrm{X}(\mathrm{t} \rightarrow \infty, \lambda>0)^{\sim} \mathrm{xe}^{|\lambda| \bullet t}$ \\
\hline
\end{tabular}

The speeds of flow toward or away from the selected fixed point for Cylindrical RF network antennas system currents and currents derivative respect to time are

$$
\begin{aligned}
& \frac{d Y(t)}{d t}=\lim _{\Delta t \rightarrow 0} \frac{Y(t+\Delta t)-Y(t)}{\Delta t}=\lim _{\Delta t \rightarrow 0} \frac{Y^{(i)}+y \cdot e^{\lambda \cdot(t+\Delta t)}-\left[Y^{(i)}+y \cdot e^{\lambda \cdot t}\right]}{\Delta t} \\
& =\lim _{\Delta t \rightarrow 0} \frac{y \cdot e^{\lambda \cdot t} \cdot\left[e^{\lambda \cdot \Delta t}-1\right]}{\Delta t} \stackrel{e^{\lambda \cdot \Delta \cdot} \approx 1+\lambda \cdot \Delta t}{\longrightarrow} \lim _{\Delta t \rightarrow 0} \frac{y \cdot e^{\lambda \cdot t} \cdot[1+\lambda \cdot \Delta t-1]}{\Delta t}=\lambda \cdot y \cdot e^{\lambda \cdot t} \\
& \frac{d X(t)}{d t}=\lim _{\Delta t \rightarrow 0} \frac{X(t+\Delta t)-X(t)}{\Delta t}=\lim _{\Delta t \rightarrow 0} \frac{X^{(i)}+x \cdot e^{\lambda \cdot(t+\Delta t)}-\left[X^{(i)}+x \cdot e^{\lambda \cdot t}\right]}{\Delta t} \\
& =\lim _{\Delta t \rightarrow 0} \frac{x \cdot e^{\lambda \cdot t} \cdot\left[e^{\lambda \cdot \Delta t}-1\right]}{\Delta t} e^{e^{\lambda \cdot \Delta} \approx 1+\lambda \cdot \Delta t} \rightarrow \lim _{\Delta t \rightarrow 0} \frac{x \cdot e^{\lambda \cdot t} \cdot[1+\lambda \cdot \Delta t-1]}{\Delta t}=\lambda \cdot x \cdot e^{\lambda \cdot t}
\end{aligned}
$$

and the time derivative of the above equations:

$$
\begin{gathered}
\frac{d Y(t)}{d t}=y \cdot \lambda \cdot e^{\lambda \cdot t} ; \frac{d X(t)}{d t}=x \cdot \lambda \cdot e^{\lambda \cdot t} \\
\frac{d Y\left(t-\tau_{1}\right)}{d t}=y \cdot \lambda \cdot e^{\lambda \cdot\left(t-\tau_{1}\right)}=y \cdot \lambda \cdot e^{\lambda \cdot t} \cdot e^{-\tau_{1} \cdot \lambda} \\
\frac{d X\left(t-\tau_{2}\right)}{d t}=x \cdot \lambda \cdot e^{\lambda \cdot\left(t-\tau_{2}\right)}=x \cdot \lambda \cdot e^{\lambda \cdot t} \cdot e^{-\tau_{2} \cdot \lambda}
\end{gathered}
$$

First we take the Cylindrical RF network antennas $(\mathrm{Y})$ differential equation: $\frac{d Y}{d t}=X \quad$ and adding to it coordinates $[\mathrm{YX}]$ arbitrarily small increments of exponential form $[y x] \cdot e^{\lambda \cdot t}$ and retaining the first order terms in $y, x$.

$$
\lambda \cdot y \cdot e^{\lambda \cdot t}=X^{(i)}+x \cdot e^{\lambda \cdot t} ; X^{(i=0)}=0 ; \lambda_{1}=\frac{x}{y} \approx 1>0
$$

Second we take the Cylindrical RF network antennas $(\mathrm{X})$ differential equation: $\frac{d Y}{d t}=X$ and adding to it coordinates $[\mathrm{YX}]$ arbitrarily small increments of exponential form $[y x] \cdot e^{\lambda \cdot t}$ and retaining the first order terms in $y, x$.

$$
\lambda \cdot x \cdot e^{\lambda \cdot t}=\frac{-1}{L \cdot C} \cdot\left[Y^{(i)}+y \cdot e^{\lambda \cdot t}\right] ; Y^{(i=0)}=0 ; \lambda_{2}=\left.\frac{-1}{L \cdot C} \cdot \frac{y}{x}\right|_{\frac{y}{x}}=\frac{-1}{L \cdot C}<0
$$

we have saddle fixed point otherwise it is unstable node (both eigenvalues are positive). We define $Y\left(t-\tau_{1}\right)=Y^{(i)}+y \cdot e^{\lambda \cdot\left(t-\tau_{1}\right)} X\left(t-\tau_{2}\right)=X^{(i)}+x \cdot e^{\lambda \cdot\left(t-\tau_{2}\right)}$ then we get two delayed differential equations 
respect to adding to it coordinates $[\mathrm{Y} X]$ arbitrarily small increments of exponential form $[y x] \cdot e^{\lambda \cdot t} \cdot \operatorname{In}$ the equilibrium points: $Y^{(0)}=0 ; X^{(0)}=0$

$$
\begin{aligned}
& \lambda \cdot y \cdot e^{\lambda \cdot t}=X^{(0)}+x \cdot e^{\lambda \cdot\left(t-\tau_{2}\right)} ; X^{(0)}=0 \Rightarrow \lambda \cdot y=x \cdot e^{-\lambda \cdot \tau_{2}} \\
& \lambda \cdot x \cdot e^{\lambda \cdot t}=\frac{-1}{L \cdot C} \cdot\left[Y^{(0)}+y \cdot e^{\lambda \cdot\left(t-\tau_{1}\right)}\right] ; Y^{(0)}=0 \Rightarrow \\
& \lambda \cdot x=\frac{-1}{L \cdot C} \cdot y \cdot e^{-\lambda \cdot \tau_{1}}
\end{aligned}
$$

We get the following set of eigenvalues equations:

$$
-\lambda \cdot y+x \cdot e^{-\lambda \cdot \tau_{2}}=0 ; \frac{-1}{L \cdot C} \cdot y \cdot e^{-\lambda \cdot \tau_{1}}-\lambda \cdot x=0
$$

The small increments Jacobian of our Cylindrical RF network antennas.

$$
\begin{gathered}
\left(\begin{array}{cc}
-\lambda & e^{-\lambda \cdot \tau_{2}} \\
\frac{-1}{L \cdot C} \cdot e^{-\lambda \cdot \tau_{1}} & -\lambda
\end{array}\right) \cdot\left(\begin{array}{l}
y \\
x
\end{array}\right)=\left(\begin{array}{l}
0 \\
0
\end{array}\right) \\
A-\lambda \cdot I=\left(\begin{array}{cc}
-\lambda & e^{-\lambda \cdot \tau_{2}} \\
\frac{-1}{L \cdot C} \cdot e^{-\lambda \cdot \tau_{1}} & -\lambda
\end{array}\right) ; \operatorname{det}|A-\lambda \cdot I|=0 \\
D\left(\lambda, \tau_{1}, \tau_{2}\right)=\lambda^{2}+\frac{1}{L \cdot C} \cdot e^{-\lambda \cdot \tau_{1}} \cdot e^{-\lambda \cdot \tau_{2}}
\end{gathered}
$$

We have three stability analysis cases: $\tau_{1}=\tau ; \tau_{2}=0$ or $\tau_{2}=\tau ; \tau_{1}=0$ or $\tau_{1}=\tau_{2}=\tau$ otherwise $\tau_{1} \neq \tau_{2}$ We need to get characteristics equations as all above stability analysis cases. We study the occurrence of any possible stability switching resulting from the increase of value of the time delay $\tau$ for the general characteristic equation $D(\lambda, \tau)$

$$
D(\lambda, \tau)=P_{n}(\lambda, \tau)+Q_{m}(\lambda, \tau) \cdot e^{-\lambda \tau}
$$

The expression for $P_{n}(\lambda, \tau)$ is

$$
\begin{aligned}
& P_{n}(\lambda, \tau)=\sum_{k=0}^{n} P_{k}(\tau) \cdot \lambda^{k}=P_{0}(\tau)+P_{1}(\tau) \cdot \lambda+P_{2}(\tau) \cdot \lambda^{2} \\
& +P_{3}(\tau) \cdot \lambda^{3}+\ldots \ldots . .
\end{aligned}
$$

The expression for $Q_{m}(\lambda, \tau)$ is

$$
Q_{m}(\lambda, \tau)=\sum_{k=0}^{m} q_{k}(\tau) \cdot \lambda^{k}=q_{0}(\tau)+q_{1}(\tau) \cdot \lambda+q_{2}(\tau) \cdot \lambda^{2}+\ldots \ldots
$$




\section{Cylindrical RF Network Antennas System Second Order Characteristic}

Equation $\tau_{1}=\tau ; \tau_{2}=0 \& \tau_{1}=0 ; \tau_{2}=\tau$

The first case we analyze is when there is delay in Cylindrical RF network antennas leg's current and no delay in antennas leg's current derivative or opposite [4] [5].

$$
\begin{gathered}
D\left(\lambda, \tau_{1}=0, \tau_{2}\right)=\lambda^{2}+\frac{1}{L \cdot C} \cdot e_{\tau_{2}=\tau}^{-\lambda \cdot \tau_{2}}=\lambda^{2}+\frac{1}{L \cdot C} \cdot e^{-\lambda \cdot \tau} \\
D\left(\lambda, \tau_{1}, \tau_{2}=0\right)=\lambda^{2}+\left.\frac{1}{L \cdot C} \cdot e^{-\lambda \cdot \tau_{1}}\right|_{\tau_{1}=\tau}=\lambda^{2}+\frac{1}{L \cdot C} \cdot e^{-\lambda \cdot \tau} \\
D(\lambda, \tau)=P_{n}(\lambda, \tau)+Q_{m}(\lambda, \tau) \cdot e^{-\lambda \tau}
\end{gathered}
$$

The expression for $P_{n}(\lambda, \tau)$ is

$$
\begin{aligned}
& P_{n}(\lambda, \tau)=\sum_{k=0}^{n} P_{k}(\tau) \cdot \lambda^{k}=P_{0}(\tau)+P_{1}(\tau) \cdot \lambda+P_{2}(\tau) \cdot \lambda^{2} \\
& =\lambda^{2} ; P_{2}(\tau)=1 ; P_{1}(\tau)=0 ; P_{0}(\tau)=0
\end{aligned}
$$

The expression for $Q_{m}(\lambda, \tau)$ is

$$
Q_{m}(\lambda, \tau)=\sum_{k=0}^{m} q_{k}(\tau) \cdot \lambda^{k}=q_{0}(\tau)=\frac{1}{L \cdot C}
$$

Our Cylindrical RF network antennas system second order characteristic equation:

$$
\begin{aligned}
& D(\lambda, \tau)=\lambda^{2}+a(\tau) \cdot \lambda+b(\tau) \cdot \lambda \cdot e^{-\lambda \cdot \tau}+c(\tau)+d(\tau) \cdot e^{-\lambda \cdot \tau} \\
& \text { Then } a(\tau)=0 ; \mathrm{b}(\tau)=0 ; \mathrm{c}(\tau)=0 ; \mathrm{d}(\tau)=\frac{1}{L \cdot C}
\end{aligned}
$$

$\tau \in R_{+0}$ and $a(\tau), \mathrm{b}(\tau), \mathrm{c}(\tau), \mathrm{d}(\tau): R_{+0} \rightarrow R$ are differentiable functions of class $C^{1}\left(R_{+0}\right)$ such that $\mathrm{c}(\tau)+\mathrm{d}(\tau)=\frac{1}{L \cdot C} \neq 0$ for all $\tau \in R_{+0}$ and for any $\tau, \mathrm{b}(\tau), \mathrm{d}(\tau)$ are not simultaneously zero. We have

$$
\begin{gathered}
P(\lambda, \tau)=P_{n}(\lambda, \tau)=\lambda^{2}+a(\tau) \cdot \lambda+c(\lambda)=\lambda^{2} \\
Q(\lambda, \tau)=Q_{m}(\lambda, \tau)=b(\tau) \cdot \lambda+d(\tau)=\frac{1}{L \cdot C}
\end{gathered}
$$

we assume that $P_{n}(\lambda, \tau)=P_{n}(\lambda)$ and $Q_{m}(\lambda, \tau)=Q_{m}(\lambda)$ can't have common imaginary roots. That is for any real number $\omega$;

$$
p_{n}(\lambda=i \cdot \omega, \tau)+Q_{m}(\lambda=i \cdot \omega, \tau) \neq 0
$$




$$
\begin{gathered}
-\omega^{2}+\frac{1}{L \cdot C} \neq 0 \\
F(\omega, \tau)=\omega^{4}-\frac{1}{(L \cdot C)^{2}} \begin{array}{l}
F(\omega, \tau)=|P(i \cdot \omega, \tau)|^{2}-|Q(i \cdot \omega, \tau)|^{2} \\
=\left(c-\omega^{2}\right)^{2}+\omega^{2} \cdot a^{2}-\left(\omega^{2} \cdot b^{2}+d^{2}\right)
\end{array}
\end{gathered}
$$

Hence

$$
F(\omega, \tau)=0 \text { implies } \omega^{4}-\frac{1}{(L \cdot C)^{2}}=0
$$

And its roots are given by

$$
\begin{gathered}
\omega_{+}^{2}=\frac{1}{2} \cdot\left\{\left(b^{2}+2 \cdot c-a^{2}\right)+\sqrt{\Delta}\right\}=\frac{\sqrt{\Delta}}{2} \\
\omega_{-}^{2}=\frac{1}{2} \cdot\left\{\left(b^{2}+2 \cdot c-a^{2}\right)-\sqrt{\Delta}\right\}=-\frac{\sqrt{\Delta}}{2} \\
\Delta=\left(b^{2}+2 \cdot c-a^{2}\right)-4 \cdot\left(c^{2}-d^{2}\right)=\frac{4}{L^{2} \cdot C^{2}}
\end{gathered}
$$

Therefore the following holds:

$$
2 \cdot \omega_{+/-}^{2}-\left(b^{2}+2 \cdot c-a^{2}\right)= \pm \sqrt{\Delta} ; 2 \cdot \omega_{+/-}^{2}= \pm \sqrt{\Delta}
$$

Furthermore $P_{R}(i \cdot \omega, \tau)=c(\tau)-\omega^{2}(\tau)=-\omega^{2}(\tau)$

$$
\begin{gathered}
P_{I}(i \cdot \omega, \tau)=\omega(\tau) \cdot a(\tau)=0 ; Q_{R}(i \cdot \omega, \tau)=d(\tau)=\frac{1}{L \cdot C} \\
Q_{I}(i \cdot \omega, \tau)=\omega(\tau) \cdot b(\tau)=0 \text { hence }
\end{gathered}
$$

$$
\sin \theta(\tau)=\frac{-P_{R}(i \cdot \omega, \tau) \cdot Q_{I}(i \cdot \omega, \tau)+P_{I}(i \cdot \omega, \tau) \cdot Q_{R}(i \cdot \omega, \tau)}{|Q(i \cdot \omega, \tau)|^{2}}
$$

$$
\cos \theta(\tau)=-\frac{P_{R}(i \cdot \omega, \tau) \cdot Q_{R}(i \cdot \omega, \tau)+P_{I}(i \cdot \omega, \tau) \cdot Q_{I}(i \cdot \omega, \tau)}{|Q(i \cdot \omega, \tau)|^{2}}
$$

$$
\begin{gathered}
\sin \theta(\tau)=\frac{-\left(c-\omega^{2}\right) \cdot \omega \cdot b+\omega \cdot a \cdot d}{\omega^{2} \cdot b^{2}+d^{2}}=0 \\
\cos \theta(\tau)=-\frac{\left(c-\omega^{2}\right) \cdot d+\omega^{2} \cdot a \cdot b}{\omega^{2} \cdot b^{2}+d^{2}}=\omega^{2} \cdot L \cdot C
\end{gathered}
$$




$$
\begin{aligned}
& \text { Which jointly with } \omega^{4}-\frac{1}{(L \cdot C)^{2}}=0 \\
& \text { Defines the maps } S_{n}(\tau)=\tau-\tau_{n}(\tau) ; \tau \in \mathrm{I}, \mathrm{n} \in \mathbb{N}_{0}
\end{aligned}
$$

That are continuous and differentiable in $\tau$ based on Lema 1.1 (see Appendix A). Hence we use theorem 1.2 (see Appendix B). This prove the theorem 1.3 (see Appendix C) and theorem 1.4 (see Appendix D).

Remark: $a, b, c, d$ parameters are independent of delay parameter $\tau$ even we use $a(\tau), \mathrm{b}(\tau), \mathrm{c}(\tau), \mathrm{d}(\tau)$.

\section{Cylindrical RF Network Antennas System Second Order Characteristic$$
\text { Equation } \tau_{1}=\tau ; \tau_{2}=\tau
$$

The second case we analyze is when there is delay both in Cylindrical RF network antennas leg's current and current time derivative [4] [5].

$$
\begin{aligned}
& D\left(\lambda, \tau_{1}=\tau, \tau_{2}=\tau\right)=\lambda^{2}+\frac{1}{L \cdot C} \cdot e^{-\lambda \cdot \tau} \cdot e^{-\lambda \cdot \tau} \\
& D(\lambda, \tau)=P_{n}(\lambda, \tau)+Q_{m}(\lambda, \tau) \cdot e^{-\lambda \tau}
\end{aligned}
$$

The expression for $P_{n}(\lambda, \tau)$ is

$$
\begin{aligned}
& P_{n}(\lambda, \tau)=\sum_{k=0}^{n} P_{k}(\tau) \cdot \lambda^{k}=P_{0}(\tau)+P_{1}(\tau) \cdot \lambda+P_{2}(\tau) \cdot \lambda^{2}=\lambda^{2} \\
& P_{2}(\tau)=1 ; P_{1}(\tau)=0 ; P_{0}(\tau)=0
\end{aligned}
$$

The expression for

$$
Q_{m}(\lambda, \tau) ; Q_{m}(\lambda, \tau)=\sum_{k=0}^{m} q_{k}(\tau) \cdot \lambda^{k}=\frac{1}{L \cdot C} \cdot e^{-\lambda \cdot \tau}
$$

Taylor expansion: $e^{-\lambda \tau} \approx 1-\lambda \cdot \tau+\frac{\lambda^{2} \cdot \tau^{2}}{2}$ since we need

$\mathrm{n}>\mathrm{m}[\mathrm{BK}]$ analysis we choose $e^{-\lambda \tau} \approx 1-\lambda \cdot \tau$ then we get

$$
\begin{aligned}
& Q_{m}(\lambda, \tau)=\sum_{k=0}^{m} q_{k}(\tau) \cdot \lambda^{k}=\frac{1}{L \cdot C} \cdot(1-\lambda \cdot \tau)=\frac{1}{L \cdot C}-\frac{1}{L \cdot C} \cdot \lambda \cdot \tau \\
& q_{0}(\tau, \lambda)=\frac{1}{L \cdot C} ; q_{1}(\tau)=-\frac{1}{L \cdot C} \cdot \tau ; q_{2}(\tau)=0
\end{aligned}
$$

Our Cylindrical RF network antennas system second order characteristic equation:

$$
D(\lambda, \tau)=\lambda^{2}+a(\tau) \cdot \lambda+b(\tau) \cdot \lambda \cdot e^{-\lambda \cdot \tau}+c(\tau)+d(\tau) \cdot e^{-\lambda \cdot \tau}
$$

Then

$$
a(\tau)=0 ; b(\tau)=\frac{-1}{L \cdot C} \cdot \tau ; c(\tau)=0 ; d(\tau)=\frac{1}{L \cdot C}
$$


And in the same manner like our previous case analysis:

$$
\begin{aligned}
& P(\lambda, \tau)=P_{n}(\lambda, \tau)=\lambda^{2} \\
& Q(\lambda, \tau)=Q_{m}(\lambda, \tau)=\frac{1}{L \cdot C}-\frac{1}{L \cdot C} \cdot \lambda \cdot \tau
\end{aligned}
$$

we assume that $P_{n}(\lambda, \tau)=P_{n}(\lambda)$ and $Q_{m}(\lambda, \tau)$ can't have common imaginary roots. That is for any real number

$$
\begin{aligned}
& \omega ; p_{n}(\lambda=i \cdot \omega, \tau)+Q_{m}(\lambda=i \cdot \omega, \tau) \neq 0 \\
& -\omega^{2}-i \cdot \omega \cdot \frac{1}{L \cdot C} \cdot \tau+\frac{1}{L \cdot C} \neq 0 \\
& F(\omega, \tau)=\left.P(i \cdot \omega, \tau)\right|^{2}-|Q(i \cdot \omega, \tau)|^{2} ; P(i \cdot \omega, \tau)=-\omega^{2} \\
& P_{R}(i \cdot \omega, \tau)=-\omega^{2} ; P_{I}(i \cdot \omega, \tau)=0 \\
& Q(\lambda=i \cdot \omega, \tau)=-i \cdot \omega \cdot \frac{1}{L \cdot C} \cdot \tau+\frac{1}{L \cdot C} \\
& Q_{I}(\lambda=i \cdot \omega, \tau)=-\omega \cdot \frac{1}{L \cdot C} \cdot \tau ; Q_{R}(\lambda=i \cdot \omega, \tau)=\frac{1}{L \cdot C} \\
& |P(i \cdot \omega, \tau)|^{2}=P_{I}^{2}+P_{R}^{2} ;|Q(i \cdot \omega, \tau)|^{2}=Q_{I}^{2}+Q_{R}^{2} \\
& |P(i \cdot \omega, \tau)|^{2}=P_{I}^{2}+P_{R}^{2}=\omega^{4} \\
& |Q(i \cdot \omega, \tau)|^{2}=\omega^{2} \cdot \frac{\tau^{2}}{(L \cdot C)^{2}}+\frac{1}{(L \cdot C)^{2}} \\
& F(\omega, \tau)=\omega^{4}-\omega^{2} \cdot \frac{\tau^{2}}{(L \cdot C)^{2}}-\frac{1}{(L \cdot C)^{2}}
\end{aligned}
$$

Hence

$$
\begin{gathered}
F(\omega, \tau)=0 \text { implies } \omega^{4}-\omega^{2} \cdot \frac{\tau^{2}}{(L \cdot C)^{2}}-\frac{1}{(L \cdot C)^{2}}=0 \\
F_{\omega}=4 \cdot \omega^{3}-2 \cdot \omega \cdot \frac{\tau^{2}}{(L \cdot C)^{2}}=2 \cdot \omega \cdot\left[2 \cdot \omega^{2}-\frac{\tau^{2}}{(L \cdot C)^{2}}\right] \\
F_{\tau}=\frac{-\omega^{2} \cdot 2 \cdot \tau}{(L \cdot C)^{2}} \\
P_{I \omega}=0 ; P_{R \omega}=-2 \cdot \omega ; Q_{I \omega}=-\frac{\tau}{L \cdot C} \\
Q_{R \omega}=0 ; P_{I \tau}=0 ; P_{R \tau}=0 ; Q_{R \tau}=0 ; Q_{I \tau}=-\frac{\omega}{L \cdot C}
\end{gathered}
$$


The expressions for $\mathrm{U}, \mathrm{V}$ can be derive easily $[\mathrm{BK}]: x=\tau$

$$
\begin{gathered}
U=\left(P_{R} \cdot P_{I \omega}-P_{I} \cdot P_{R \omega}\right)-\left(Q_{R} \cdot Q_{I \omega}-Q_{I} \cdot Q_{R \omega}\right) \\
V=\left(P_{R} \cdot P_{I x}-P_{I} \cdot P_{R x}\right)-\left(Q_{R} \cdot Q_{I x}-Q_{I} \cdot Q_{R x}\right) \\
V=\frac{\omega}{L^{2} \cdot C^{2}} ; U=\frac{\tau}{L^{2} \cdot C^{2}} \\
\omega_{\tau}=-\frac{F_{\tau}}{F_{\omega}}
\end{gathered}
$$

and we get the expression:

$$
\omega_{\tau}=-\frac{\frac{-\omega^{2} \cdot 2 \cdot \tau}{(L \cdot C)^{2}}}{2 \cdot \omega \cdot\left[2 \cdot \omega^{2}-\frac{\tau^{2}}{(L \cdot C)^{2}}\right]}=\frac{\frac{-\omega \cdot \tau}{(L \cdot C)^{2}}}{\left[2 \cdot \omega^{2}-\frac{\tau^{2}}{(L \cdot C)^{2}}\right]}
$$

Defines the maps $S_{n}(\tau)=\tau-\tau_{n}(\tau) ; \tau \in \mathrm{I}, \mathrm{n} \in \mathbb{N}_{0}$

Defines the maps $S_{n}(\tau)=\tau-\tau_{n}(\tau) ; \tau \in \mathrm{I}, \mathrm{n} \in \mathbb{N}_{0}$

That are continuous and differentiable in $\tau$ based on Lema 1.1 (see Appendix A). Hence we use theorem 1.2 (see Appendix B). This prove the theorem 1.3 (see Appendix C) and theorem 1.4 (see Appendix D).

Remark: Taylor approximation for $e^{-\lambda \tau} \approx 1-\lambda \cdot \tau$ gives us Good stability analysis approximation only for restricted delay time interval.

\section{Cylindrical RF Network Antennas System Stability Analysis under Delayed Variables in Time $\tau_{1}=\tau ; \tau_{2}=\tau$}

Our Cylindrical RF network antennas homogeneous system for $\mathrm{y}, \mathrm{x}$ leads to a characteristic equation for the eigenvalue $\lambda$ having the form $P(\lambda)+Q(\lambda) \cdot e^{-\lambda \cdot \tau}=0 ;$ second case $\tau_{1}=\tau ; \tau_{2}=\tau$

$$
D\left(\lambda, \tau_{1}=\tau, \tau_{2}=\tau\right)=\lambda^{2}+\frac{1}{L \cdot C} \cdot e^{-\lambda \cdot \tau} \cdot e^{-\lambda \cdot \tau}
$$

We estimate $e^{-\lambda \tau} \approx 1-\lambda \cdot \tau$

$$
\begin{aligned}
& D\left(\lambda, \tau_{1}=\tau, \tau_{2}=\tau\right)=\lambda^{2}+\frac{1}{L \cdot C} \cdot(1-\lambda \cdot \tau) \cdot e^{-\lambda \cdot \tau} \\
& D\left(\lambda, \tau_{1}=\tau, \tau_{2}=\tau\right)=\lambda^{2}+\left(-\lambda \cdot \frac{1}{L \cdot C} \cdot \tau+\frac{1}{L \cdot C}\right) \cdot e^{-\lambda \cdot \tau}
\end{aligned}
$$

We use different parameters terminology from our last characteristics parameters definition: 


$$
k \rightarrow j ; p_{k}(\tau) \rightarrow a_{j} ; q_{k}(\tau) \rightarrow c_{j} ; n=2 ; m=1
$$

Additionally $P_{n}(\lambda, \tau) \rightarrow P(\lambda) ; Q_{m}(\lambda, \tau) \rightarrow Q(\lambda)$

$$
\text { then } \begin{aligned}
P(\lambda) & =\sum_{j=0}^{2} a_{j} \cdot \lambda^{j} \text { and } Q(\lambda)=\sum_{j=0}^{1} c_{j} \cdot \lambda^{j} . \\
P(\lambda) & =\lambda^{2} ; Q(\lambda, \tau)=-\lambda \cdot \frac{1}{L \cdot C} \cdot \tau+\frac{1}{L \cdot C}
\end{aligned}
$$

$n, m \in \mathbb{N}_{0}, n>m$ and $a_{j}, c_{j}: \mathrm{R}_{+0} \rightarrow R$ are continuous and differentiable function of $\tau$ such that $a_{0}+c_{0} \neq 0$. In the following "-"denotes complex and conjugate. $P(\lambda), Q(\lambda)$

Are analytic functions in $\lambda$ and differentiable in $\tau$.

And the coefficients: $\left\{a_{j}(C, L), c_{j}(C, L, \tau)\right\} \in \mathbb{R}$ depend on Cylindrical RF network antennas $\mathrm{C}, \mathrm{L}, \tau$ values.

$$
a_{0}=0, a_{1}=0, a_{2}=1 ; c_{0}=\frac{1}{L \cdot C}, c_{1}=-\frac{1}{L \cdot C} \cdot \tau
$$

Unless strictly necessary, the designation of the variation arguments $(C, L, \tau)$ will subsequently be omitted from $P, Q, a_{j}, c_{j}$. The coefficients $a_{j}, c_{j}$ are continuous, and differentiable functions of their arguments, and direct substitution shows that

$$
a_{0}+c_{0}=\frac{1}{L \cdot C} \neq 0 ; \frac{1}{L \cdot C} \neq 0 \forall C, L, \tau \in \mathbb{R}_{+} \text {i.e }
$$

$\lambda=0$ is not a root of characteristic equation. Furthermore $P(\lambda), Q(\lambda)$ are analytic function of $\lambda$ for which the following requirements of the analysis (see kuang, 1993, section 3.4) can also be verified in the present case [4] [5].

a) If $\lambda=i \cdot \omega, \omega \in \mathbb{R}$ then $P(i \cdot \omega)+Q(i \cdot \omega) \neq 0$, i.e $\mathrm{P}$ and $\mathrm{Q}$ have no common imaginary roots. This condition was verified numerically in the entire $(C, L, \tau)$ domain of interest.

b) $|\mathrm{Q}(\lambda) / P(\lambda)|$ is bounded for $|\lambda| \rightarrow \infty, \operatorname{Re} \lambda \geq 0$. No roots bifurcation from $\infty$. Indeed, in the limit

$$
|\mathrm{Q}(\lambda) / P(\lambda)|=\left|\frac{-\lambda \cdot \frac{1}{L \cdot C} \cdot \tau+\frac{1}{L \cdot C}}{\lambda^{2}}\right|
$$

c)

$$
\begin{gathered}
F(\omega)=|P(i \cdot \omega)|^{2}-|Q(i \cdot \omega)|^{2} \\
F(\omega, \tau)=\omega^{4}-\omega^{2} \cdot \frac{\tau^{2}}{(L \cdot C)^{2}}-\frac{1}{(L \cdot C)^{2}}
\end{gathered}
$$


Has at most a finite number of zeros. Indeed, this is a bi-cubic polynomial in $\omega$ (second degree in $\omega^{2}$ ).

d) Each positive root $\omega(C, L, \tau)$ of $\mathrm{F}(\omega)=0$ is continuous and differentiable with respect to $C, L, \tau$

. This condition can only be assessed numerically.

In addition, since the coefficients in $\mathrm{P}$ and $\mathrm{Q}$ are real, we have $\overline{P(-i \cdot \omega)}=P(i \cdot \omega)$, and $\overline{Q(-i \cdot \omega)}=Q(i \cdot \omega)$ thus $\lambda=i \cdot \omega, \omega>0$ may be on eigenvalue of characteristic equation. The analysis consists in identifying the roots of characteristic equation situated on the imaginary axis of the complex $\lambda$ - plane, where by increasing the parameters

$C, L$ and delay $\tau, \operatorname{Re} \lambda$ may, at the crossing ,Change its sign from (-) to (+), i.e. from stable focus $E^{(0)}\left(Y^{(0)}, X^{(0)}\right)=(0,0)$ to an unstable one, or vice versa. This feature may be further assessed by examining the sign of the partial derivatives with respect to $C, L$ and antenna parameters.

$$
\begin{aligned}
& \wedge^{-1}(C)=\left(\frac{\partial \operatorname{Re} \lambda}{\partial C}\right)_{\lambda=i \cdot \omega}, L, \tau=\text { const } \\
& \wedge^{-1}(L)=\left(\frac{\partial \operatorname{Re} \lambda}{\partial L}\right)_{\lambda=i \cdot \omega}, C, \tau=\text { const } \\
& \wedge^{-1}(\tau)=\left(\frac{\partial \operatorname{Re} \lambda}{\partial \tau}\right)_{\lambda=i \cdot \omega}, C, L, \text { where } \omega \in \mathbb{R}_{+}
\end{aligned}
$$

For the first case $\tau_{1}=\tau ; \tau_{2}=\tau$ we get the following results

$$
\begin{aligned}
& P_{R}(i \cdot \omega)=-\omega^{2} ; P_{I}(i \cdot \omega)=0 ; Q_{R}(i \cdot \omega)=\frac{1}{L \cdot C} \\
& Q_{I}(i \cdot \omega)=\frac{-\omega \cdot \tau}{L \cdot C} ; F(\omega)=0 \text { yield to } \\
& \omega^{4}-\omega^{2} \cdot \frac{\tau^{2}}{(L \cdot C)^{2}}-\frac{1}{(L \cdot C)^{2}}=0 ; \chi^{2}=\omega^{4} ; \chi=\omega^{2} \\
& \chi^{2}-\chi \cdot \frac{\tau^{2}}{(L \cdot C)^{2}}-\frac{1}{(L \cdot C)^{2}}=0 \\
& \chi=\frac{\tau^{2}}{2 \cdot(L \cdot C)^{2}} \pm \frac{1}{2} \cdot \sqrt{\frac{\tau^{4}}{(L \cdot C)^{4}}+4 \cdot \frac{1}{(L \cdot C)^{2}}} \\
& \chi=\omega^{2} \Rightarrow \omega= \pm \sqrt{\frac{\tau^{2}}{2 \cdot(L \cdot C)^{2}} \pm \frac{1}{2} \cdot \sqrt{\frac{\tau^{4}}{(L \cdot C)^{4}}+4 \cdot \frac{1}{(L \cdot C)^{2}}}} \\
& \frac{\tau^{4}}{(L \cdot C)^{4}}+4 \cdot \frac{1}{(L \cdot C)^{2}}>0
\end{aligned}
$$




$$
\text { always and additional for } \omega \in R ; \omega^{2}=\frac{\tau^{2}}{2 \cdot(L \cdot C)^{2}} \pm \frac{1}{2} \cdot \sqrt{\frac{\tau^{4}}{(L \cdot C)^{4}}+4 \cdot \frac{1}{(L \cdot C)^{2}}}
$$

And there are two options: first always exist

$$
\begin{aligned}
& \frac{\tau^{2}}{2 \cdot(L \cdot C)^{2}}+\frac{1}{2} \cdot \sqrt{\frac{\tau^{4}}{(L \cdot C)^{4}}+4 \cdot \frac{1}{(L \cdot C)^{2}}}>0 \\
& \text { Second } \frac{\tau^{2}}{2 \cdot(L \cdot C)^{2}}-\frac{1}{2} \cdot \sqrt{\frac{\tau^{4}}{(L \cdot C)^{4}}+4 \cdot \frac{1}{(L \cdot C)^{2}}}<0 \\
& \omega^{2}=\frac{1}{2} \cdot \frac{1}{L \cdot C} \cdot\left\{\frac{\tau^{2}}{L \cdot C} \pm \sqrt{\left.\frac{\tau^{4}}{(L \cdot C)^{2}}+4\right\}}\right.
\end{aligned}
$$

$\sqrt{\frac{\tau^{4}}{(L \cdot C)^{2}}+4}>\frac{\tau^{2}}{L \cdot C}$, Not exist and always negative for any Cylindrical RF network antennas overall parameters values. We choose only the (+) option (first).

$$
\text { Writing } P(\lambda)=P_{R}(\lambda)+i \cdot P_{I}(\lambda) \text { and } Q(\lambda)=Q_{R}(\lambda)+i \cdot Q_{I}(\lambda)
$$

and inserting $\lambda=i \cdot \omega$ Into Cylindrical RF network antennas characteristic equation, $\omega$ must satisfy the following:

$$
\begin{aligned}
& \sin \omega \cdot \tau=g(\omega)=\frac{-P_{R}(i \cdot \omega) \cdot Q_{I}(i \cdot \omega)+P_{I}(i \cdot \omega) \cdot Q_{R}(i \cdot \omega)}{|Q(i \cdot \omega)|^{2}} \\
& \cos \omega \cdot \tau=h(\omega)=-\frac{P_{R}(i \cdot \omega) \cdot Q_{R}(i \cdot \omega)+P_{I}(i \cdot \omega) \cdot Q_{I}(i \cdot \omega)}{|Q(i \cdot \omega)|^{2}}
\end{aligned}
$$

Where $|Q(i \cdot \omega)|^{2} \neq 0$ in view of requirement (a) above, and $(g, h) \in R$. Furthermore, it follows above $\sin \omega \cdot \tau$ and $\cos \omega \cdot \tau$ equations that, by squaring and adding the sides, $\omega$ Must be a positive root of

$$
F(\omega)=|P(i \cdot \omega)|^{2}-|Q(i \cdot \omega)|^{2}=0 .
$$

Note that $F(\omega)$ is dependent of $\tau$. Now it is important to notice that if $\tau \notin I$ (assume that $I \subseteq R_{+0}$ is the set where $\omega(\tau)$ is a positive root of $F(\omega)$ and for $\tau \notin I, \omega(\tau)$ is not define. Then for all $\tau$ in I $\omega(\tau)$ is satisfies that $F(\omega, \tau)=0$

Then there are positive $\omega(\tau)$ solutions of $F(\omega, \tau)=0$, and we analyze stability switches. For any $\tau \in I$ where $\omega(\tau)$ is a positive solution of $F(\omega, \tau)=0$, we can define the angle $\theta(\tau) \in[0,2 \cdot \pi]$ as the solution of

$$
\sin \theta(\tau)=\frac{-P_{R}(i \cdot \omega) \cdot Q_{I}(i \cdot \omega)+P_{I}(i \cdot \omega) \cdot Q_{R}(i \cdot \omega)}{|Q(i \cdot \omega)|^{2}}
$$




$$
\cos \theta(\tau)=-\frac{P_{R}(i \cdot \omega) \cdot Q_{R}(i \cdot \omega)+P_{I}(i \cdot \omega) \cdot Q_{I}(i \cdot \omega)}{|Q(i \cdot \omega)|^{2}}
$$

And the relation between the argument $\theta(\tau)$ and $\omega(\tau) \cdot \tau$ for

$\tau \in I$ must be $\omega(\tau) \cdot \tau=\theta(\tau)+n \cdot 2 \cdot \pi \quad \forall n \in \mathbb{N}_{0}$. Hence we can define the maps $\tau_{n}: I \rightarrow R_{+0}$ given by $\tau_{n}(\tau)=\frac{\theta(\tau)+n \cdot 2 \cdot \pi}{\omega(\tau)} ; n \in \mathbb{N}_{0}, \tau \in I \quad$. Let us introduce the functions $I \rightarrow R$; $S_{n}(\tau)=\tau-\tau_{n}(\tau), \tau \in I, n \in \mathbb{N}_{0}$

That are continuous and differentiable in $\tau$. In the following, the subscripts $\lambda, \omega, C, L$ and Cylindrical $\mathrm{RF}$ network antennas parameters $(L, C, \tau$ etc., $)$ indicate the corresponding partial derivatives. Let us first concentrate on $\wedge(x)$, remember in $\lambda(L, C, \tau$, etc., $)$ and $\omega(L, C, \tau$, etc., $)$, and keeping all parameters except one $(\mathrm{x})$ and $\tau$. The derivation closely follows that in reference [BK]. Differentiating Cylindrical RF network antennas characteristic equation $P(\lambda)+Q(\lambda) \cdot e^{-\lambda \cdot \tau}=0$ with respect to specific parameter $(\mathrm{x})$, and inverting the derivative, for convenience, one calculates: Remark: $x=L, C, \tau$, etc.,

$$
\begin{aligned}
& \left(\frac{\partial \lambda}{\partial x}\right)^{-1} \\
& =\frac{-P_{\lambda}(\lambda, x) \cdot Q(\lambda, x)+Q_{\lambda}(\lambda, x) \cdot P(\lambda, x)-\tau \cdot P(\lambda, x) \cdot Q(\lambda, x)}{P_{x}(\lambda, x) \cdot Q(\lambda, x)-Q_{x}(\lambda, x) \cdot P(\lambda, x)}
\end{aligned}
$$

Where $P_{\lambda}=\frac{\partial P}{\partial \lambda}, \ldots .$. etc., Substituting $\lambda=i \cdot \omega$, and Bearing i $\overline{P(-i \cdot \omega)}=P(i \cdot \omega)$,

$$
\overline{Q(-i \cdot \omega)}=Q(i \cdot \omega)
$$

Then $i \cdot P_{\lambda}(i \cdot \omega)=P_{\omega}(i \cdot \omega)$ and $i \cdot Q_{\lambda}(i \cdot \omega)=Q_{\omega}(i \cdot \omega)$ and that on the surface $|P(i \cdot \omega)|^{2}=|Q(i \cdot \omega)|^{2}$, one obtains

$$
\begin{aligned}
& \left.\left(\frac{\partial \lambda}{\partial x}\right)^{-1}\right|_{\lambda=i \omega} \\
& =\left(\frac{i \cdot P_{\omega}(i \cdot \omega, x) \cdot \overline{P(i \cdot \omega, x)}+i \cdot Q_{\lambda}(i \cdot \omega, x) \cdot \overline{Q(\lambda, x)}-\tau \cdot|P(i \cdot \omega, x)|^{2}}{P_{x}(i \cdot \omega, x) \cdot \overline{P(i \cdot \omega, x)}-Q_{x}(i \cdot \omega, x) \cdot \overline{Q(i \cdot \omega, x)}}\right)
\end{aligned}
$$

Upon separating into real and imaginary parts, with

$$
\begin{aligned}
& P=P_{R}+i \cdot P_{I} ; Q=Q_{R}+i \cdot Q_{I} ; P_{\omega}=P_{R \omega}+i \cdot P_{I \omega} \\
& Q_{\omega}=Q_{R \omega}+i \cdot Q_{I \omega} ; P_{x}=P_{R x}+i \cdot P_{I x} ; Q_{x}=Q_{R x}+i \cdot Q_{I x}
\end{aligned}
$$

$P^{2}=P_{R}^{2}+P_{I}^{2}$. When $(\mathrm{x})$ can be any Cylindrical RF network antennas parameters $\mathrm{L}, \mathrm{C}$, And time delay $\tau$ etc,. Where for convenience, we have dropped the arguments $(i \cdot \omega, x)$, and where

$$
\begin{aligned}
& F_{\omega}=2 \cdot\left[\left(P_{R \omega} \cdot P_{R}+P_{I \omega} \cdot P_{I}\right)-\left(Q_{R \omega} \cdot Q_{R}+Q_{I \omega} \cdot Q_{I}\right)\right] \\
& F_{x}=2 \cdot\left[\left(P_{R x} \cdot P_{R}+P_{I x} \cdot P_{I}\right)-\left(Q_{R x} \cdot Q_{R}+Q_{I x} \cdot Q_{I}\right)\right]
\end{aligned}
$$


$\omega_{x}=-F_{x} / F_{\omega}$. We define $\mathrm{U}$ and $\mathrm{V}$ :

$$
\begin{gathered}
U=\left(P_{R} \cdot P_{I \omega}-P_{I} \cdot P_{R \omega}\right)-\left(Q_{R} \cdot Q_{I \omega}-Q_{I} \cdot Q_{R \omega}\right) \\
V=\left(P_{R} \cdot P_{I x}-P_{I} \cdot P_{R x}\right)-\left(Q_{R} \cdot Q_{I x}-Q_{I} \cdot Q_{R x}\right)
\end{gathered}
$$

We choose our specific parameter as time delay $x=\tau$.

$$
\begin{aligned}
& V=\frac{\omega}{L^{2} \cdot C^{2}} ; U=\frac{\tau}{L^{2} \cdot C^{2}} ; P^{2}=\omega^{4} ; F_{\tau}=\frac{-\omega^{2} \cdot 2 \cdot \tau}{(L \cdot C)^{2}} \\
& P_{R}(\omega, \tau)=-\omega^{2} ; P_{I}(\omega, \tau)=0 \\
& Q_{I}(\omega, \tau)=-\frac{\omega \cdot \tau}{L \cdot C} ; Q_{R}(\omega, \tau)=\frac{1}{L \cdot C} \\
& P_{I \tau}=0 ; P_{R \tau}=0 ; Q_{R \tau}=0 ; Q_{I \tau}=-\frac{\omega}{L \cdot C} \Rightarrow V \neq 0 \\
& \frac{\partial F}{\partial \omega}=F_{\omega}=4 \cdot \omega^{3}-2 \cdot \omega \cdot \frac{\tau^{2}}{(L \cdot C)^{2}} \\
& \frac{\partial F}{\partial \omega}=2 \cdot \omega \cdot\left[2 \cdot \omega^{2}-\frac{\tau^{2}}{(L \cdot C)^{2}}\right] ; F(\omega, \tau)=0
\end{aligned}
$$

And differentiating with respect to $\tau$ and we get

$$
\begin{gathered}
F_{\omega} \cdot \frac{\partial \omega}{\partial \tau}+F_{\tau}=0 ; \tau \in I \Rightarrow \omega_{\tau}=\frac{\partial \omega}{\partial \tau}=-\frac{F_{\tau}}{F_{\omega}} \\
\frac{\partial \omega}{\partial \tau}=\frac{\frac{\omega \cdot \tau}{(L \cdot C)^{2}}}{\left[2 \cdot \omega^{2}-\frac{\tau^{2}}{(L \cdot C)^{2}}\right]} ; \wedge^{-1}(\tau)=\left(\frac{\partial \operatorname{Re} \lambda}{\partial \tau}\right)_{\lambda=i \cdot \omega} \\
\omega_{\tau}=\frac{\partial \omega}{\partial \tau}=\frac{\omega \cdot \tau}{\left[2 \cdot \omega^{2} \cdot(L \cdot C)^{2}-\tau^{2}\right]} \\
\wedge^{-1}(\tau)=\operatorname{Re}\left\{\frac{-2 \cdot\left[U+\tau \cdot|P|^{2}\right]+i \cdot F_{\omega}}{F_{\tau}+i \cdot 2 \cdot\left[V+\omega \cdot|P|^{2}\right]}\right\} \\
=\operatorname{Re}\left\{\frac{-\tau \cdot\left[\frac{1}{L^{2} \cdot C^{2}}+\omega^{4}\right]+i \cdot \omega \cdot\left[2 \cdot \omega^{2}-\frac{\tau^{2}}{(L \cdot C)^{2}}\right.}{\frac{-\omega^{2} \cdot \tau}{(L \cdot C)^{2}}+i \cdot \omega \cdot\left[\frac{1}{L^{2} \cdot C^{2}}+\omega^{4}\right]}\right\} \\
\operatorname{sign}\left\{\wedge^{-1}(\tau)\right\}=\operatorname{sign}\left\{\left(\frac{\partial \operatorname{Re} \lambda}{\partial \tau}\right)_{\lambda=i \cdot \omega}\right\}
\end{gathered}
$$




$$
\begin{gathered}
\operatorname{sign}\left\{\wedge^{-1}(\tau)\right\}=\operatorname{sign}\left\{F_{\omega}\right\} \cdot \operatorname{sign}\left\{\tau \cdot \frac{\partial \omega}{\partial \tau}+\omega+\frac{U \cdot \frac{\partial \omega}{\partial \tau}+V}{|P|^{2}}\right\} \\
\operatorname{sign}\left\{\wedge^{-1}(\tau)\right\}=\operatorname{sign}\left\{2 \cdot \omega \cdot\left[2 \cdot \omega^{2}-\frac{\tau^{2}}{(L \cdot C)^{2}}\right]\right\} \\
\cdot \operatorname{sign}\left\{\tau \cdot\left[\frac{\frac{\omega \cdot \tau}{(L \cdot C)^{2}}}{\left[2 \cdot \omega^{2}-\frac{\tau^{2}}{(L \cdot C)^{2}}\right]}\right]+\omega\right. \\
\left.+\frac{\frac{\tau \cdot \tau}{L^{2} \cdot C^{2}} \cdot\left[\frac{[L \cdot C)^{2}}{\left[2 \cdot \omega^{2}-\frac{\tau^{2}}{(L \cdot C)^{2}}\right]}\right]+\frac{\omega}{L^{2} \cdot C^{2}}}{\omega^{4}}\right\}
\end{gathered}
$$

We define new variables: $\psi_{1}, \psi_{2}, \psi_{3}$

$$
\begin{gathered}
\psi_{1}(\omega, \tau, L, C)=2 \cdot \omega \cdot\left[2 \cdot \omega^{2}-\frac{\tau^{2}}{(L \cdot C)^{2}}\right] \\
\psi_{2}(\omega, \tau, L, C)=\tau \cdot\left[\frac{\frac{\omega \cdot \tau}{(L \cdot C)^{2}}}{\left[2 \cdot \omega^{2}-\frac{\tau^{2}}{(L \cdot C)^{2}}\right]}\right] \\
\psi_{3}(\omega, \tau, L, C)=\frac{\frac{\tau}{L^{2} \cdot C^{2}} \cdot\left[\frac{\omega \cdot \tau}{(L \cdot C)^{2}}\right]+\frac{\omega}{L^{2} \cdot C^{2}}}{\left[2 \cdot \omega^{2}-\frac{\tau^{2}}{(L \cdot C)^{2}}\right]}
\end{gathered}
$$

$\operatorname{sign}\left\{\wedge^{-1}(\tau)\right\}=\operatorname{sign}\left[\psi_{1}\right] \cdot \operatorname{sign}\left[\psi_{2}+\omega+\psi_{3}\right]$

We check the sign of $\wedge^{-1}(\tau)$ according the following rule:

\begin{tabular}{|c|c|c|}
\hline $\operatorname{sign}\left[F_{\omega}\right]$ & $\operatorname{sign}\left[\frac{V+\omega_{\tau} \cdot U}{P^{2}}+\omega+\omega_{\tau} \cdot \tau\right]$ & $\operatorname{sign}\left[\wedge^{-1}(\tau)\right]$ \\
\hline$+/-$ & $+/-$ & + \\
\hline$+/-$ & $-/+$ & - \\
\hline
\end{tabular}

Table 2. Cylindrical RF network antennas system stability switching criteria.

If $\operatorname{sign}\left[\Lambda^{-1}(\tau)\right]>0$ then the crossing proceeds from $(-)$ to $(+)$ respectively (stable to unstable). If $\operatorname{sign}\left[\Lambda^{-1}(\tau)\right]$ $<0$ then the crossing proceeds from $(+)$ to $(-)$ respectively (unstable to stable). Anyway the stability switching can occur only for specific $\omega, \tau$. Since it is a very complex function, we recommend to solve it numerically rather than analytic. We plot the stability switch diagram based on different delay values of our Cylindrical RF network antennas system. 


$$
D\left(\lambda, \tau_{1}=\tau_{2}=\tau\right)=\lambda^{2}+\frac{1}{L \cdot C} \cdot e^{-\lambda \cdot \tau}-\lambda \cdot \frac{\tau}{L \cdot C} \cdot e^{-\lambda \cdot \tau}
$$

Taylor expansion: $e^{-\lambda \tau} \approx 1-\lambda \cdot \tau+\frac{\lambda^{2} \cdot \tau^{2}}{2}$ since we need

$\mathrm{n}>\mathrm{m}[\mathrm{BK}]$ analysis we choose $e^{-\lambda \tau} \approx 1-\lambda \cdot \tau$ then we get

Our Cylindrical RF network antennas system second order characteristic equation:

$$
\begin{aligned}
& D(\lambda, \tau)=\lambda^{2}+a(\tau) \cdot \lambda+b(\tau) \cdot \lambda \cdot e^{-\lambda \cdot \tau} \\
& +c(\tau)+d(\tau) \cdot e^{-\lambda \cdot \tau} \\
& a(\tau)=0 ; b(\tau)=-\frac{\tau}{L \cdot C} ; c(\tau)=0 ; d(\tau)=\frac{1}{L \cdot C} \\
& F(\omega, \tau)=|P(i \cdot \omega, \tau)|^{2}-|Q(i \cdot \omega, \tau)|^{2}=\left(c-\omega^{2}\right)^{2} \\
& +\omega^{2} \cdot a^{2}-\left(\omega^{2} \cdot b^{2}+d^{2}\right) \\
& F(\omega, \tau)=\omega^{4}-\omega^{2} \cdot \frac{\tau^{2}}{(L \cdot C)^{2}}-\frac{1}{(L \cdot C)^{2}}
\end{aligned}
$$

Hence

$$
F(\omega, \tau)=0 \text { implies } \omega^{4}-\omega^{2} \cdot \frac{\tau^{2}}{(L \cdot C)^{2}}-\frac{1}{(L \cdot C)^{2}}=0
$$

And its roots are given by

$$
\begin{gathered}
\omega_{+}^{2}=\frac{1}{2} \cdot\left\{\left(b^{2}+2 \cdot c-a^{2}\right)+\sqrt{\Delta}\right\}=\frac{1}{2} \cdot\left\{\sqrt{\Delta}+\frac{\tau^{2}}{(L \cdot C)^{2}}\right\} \\
\omega_{-}^{2}=\frac{1}{2} \cdot\left\{\left(b^{2}+2 \cdot c-a^{2}\right)-\sqrt{\Delta}\right\}=\frac{1}{2} \cdot\left\{-\sqrt{\Delta}+\frac{\tau^{2}}{(L \cdot C)^{2}}\right\} \\
\Delta=\left(b^{2}+2 \cdot c-a^{2}\right)-4 \cdot\left(c^{2}-d^{2}\right)=\frac{\tau^{2}+4}{(L \cdot C)^{2}}
\end{gathered}
$$

Therefore the following holds:

$$
\begin{aligned}
& 2 \cdot \omega_{+--}^{2}-\left(b^{2}+2 \cdot c-a^{2}\right)= \pm \sqrt{\Delta} \\
& \sin \theta(\tau)=\frac{-P_{R}(i \cdot \omega, \tau) \cdot Q_{I}(i \cdot \omega, \tau)+P_{I}(i \cdot \omega, \tau) \cdot Q_{R}(i \cdot \omega, \tau)}{|Q(i \cdot \omega, \tau)|^{2}} \\
& \cos \theta(\tau)=-\frac{P_{R}(i \cdot \omega, \tau) \cdot Q_{R}(i \cdot \omega, \tau)+P_{I}(i \cdot \omega, \tau) \cdot Q_{I}(i \cdot \omega, \tau)}{|Q(i \cdot \omega, \tau)|^{2}}
\end{aligned}
$$




$$
\begin{array}{r}
\sin \theta(\tau)=\frac{-\left(c-\omega^{2}\right) \cdot \omega \cdot b+\omega \cdot a \cdot d}{\omega^{2} \cdot b^{2}+d^{2}}=\frac{-\omega^{3} \cdot \tau \cdot L \cdot C}{\left(\omega^{2} \cdot \tau^{2}+1\right)} \\
\cos \theta(\tau)=-\frac{\left(c-\omega^{2}\right) \cdot d+\omega^{2} \cdot a \cdot b}{\omega^{2} \cdot b^{2}+d^{2}}=\frac{\omega^{2} \cdot L \cdot C}{\left(\omega^{2} \cdot \tau^{2}+1\right)}
\end{array}
$$

We consider Cylindrical RF antenna which mounted outside a Pyrex glass tube of diameter $32 \mathrm{~cm}$ and length $50 \mathrm{~cm}$. The RF antenna consists of 16 copper $(\mathrm{Cu})$ legs equally spaced by $6.7 \mathrm{~cm}$ interconnected with capacitors of $2.47 \mathrm{nF}$. Copper leg diameter is equal to $1 \mathrm{~mm}$ and length $30 \mathrm{~cm}=300 \mathrm{~mm}$ (<Pyrex glass tube length, $50 \mathrm{~cm})$. We consider for Copper $(\mathrm{Cu})$, relative permeability is one. $f=10 \mathrm{MHz}$ is the typical testing frequency for cylindrical (birdcage) antenna. $L$ - Inductance $(\mathrm{nH}), \mathrm{I}$ - length of copper leg $(\mathrm{mm}), \mathrm{d}$ - diameter of copper leg, $f$ - testing frequency. $\mid>100 \cdot d \quad(300 \mathrm{~mm}>100 \cdot 1 \mathrm{~mm}), d^{2} \cdot f>1 \mathrm{~mm}^{2} \cdot \mathrm{MHz}$ $\left(1 \mathrm{~mm}^{2} \cdot 10 \mathrm{MHz}>1 \mathrm{~mm}^{2} \cdot \mathrm{MHz}\right) . \mathrm{L}=365.4 \mathrm{nH} . L=\frac{1}{5} \cdot l \cdot\left[\ln \left(\frac{4 \cdot l}{d}-1\right]=365.4 n H\right.$. For our stability switching analysis we choose typical Cylindrical RF network antennas parameters values (as calculated):

$C=2.47 \mathrm{nF} ; L=365.4 \mathrm{nH} ; R_{p}=100 \mathrm{ohm}$ then $\frac{1}{L \cdot C}=0.00110798 \cdot 10^{18}$. We find those $\omega, \tau$ values which fulfill $F(\omega, \tau)=0$. We ignore negative, complex, and imaginary values of $\omega$ for specific $\tau$ values. The below table gives the list. Remark: we know $F(\omega, \tau)=0$ implies it roots $\omega_{i}(\tau)$ and finding those delays values $\tau$ which $\omega_{i}$ is feasible. There are $\tau$ values, which $\omega_{i}$ are complex or imaginary numbered, then unable to analyze stability [6] [7].

\section{Cylindrical RF Network Antennas System Stability Analysis under Delayed Variables in Time $\tau_{1}=\tau ; \tau_{2}=\tau$, Results}

We find those $\omega, \tau$ values which fulfill $F(\omega, \tau)=0$. We ignore negative, complex, and imaginary values of $\omega$ for specific $\tau$ values. $\tau \in[0.001 . .10]$ and we can be express by 3D function $F(\omega, \tau)=0$.

$$
\begin{aligned}
& F(\omega, \tau)=\omega^{4}-\omega^{2} \cdot \frac{\tau^{2}}{(L \cdot C)^{2}}-\frac{1}{(L \cdot C)^{2}} \\
& F(\omega, \tau)=|P(i \cdot \omega, \tau)|^{2}-|Q(i \cdot \omega, \tau)|^{2} \\
& =\Phi_{0}+\Phi_{2} \cdot \omega^{2}+\Phi_{4} \cdot \omega^{4}=\sum_{k=0}^{2} \Phi_{2 \cdot k} \cdot \omega^{2 \cdot k} \\
& \Phi_{0}=-\frac{1}{(L \cdot C)^{2}} ; \Phi_{2}=-\frac{\tau^{2}}{(L \cdot C)^{2}} ; \Phi_{4}=1 \\
& \text { Hence } F(\omega, \tau)=0 \text { implies } \sum_{k=0}^{4} \Phi_{2 \cdot k} \cdot \omega^{2 \cdot k}=0
\end{aligned}
$$

$\Phi_{\mathrm{j}} \rightarrow \mathrm{Phi}_{\mathrm{j}}$. Running MATLAB script for $\tau$ values $(\tau \in[0.001 . .10])$ gives the following results:

MATLAB script: Tau=0.001;C=2.47*1e-9;L=365.4*1e-9;Phi0=-1/(C*L*C*L); Phi2=-(Tau*Tau)/(C*L*C*L); Phi4=1; $p=[$ Phi4 0 Phi2 0 Phi0];r=roots(p) 
Ofer Aluf; Cylindrical RF Network Antennas for Coupled Plasma Sources Copper Legs Delayed in Time System Stability Analysis, Transactions on Networks and Communications, Volume 2 No 5, Oct (2014); pp: 116-146

\begin{tabular}{|c|c|c|c|c|c|}
\hline \multicolumn{3}{|c|}{$\begin{array}{l}\text { Table 3a. Cylindrical RF network antennas systen } \\
\text { roots } \omega_{i}(\tau)\end{array}$} & \multicolumn{3}{|c|}{$\begin{array}{l}\text { Table 3b. Cylindrical RF network antennas system } \\
\text { roots } \omega_{i}(\tau)\end{array}$} \\
\hline$\tau$ & $\tau=0.01 \mathrm{sec}$ & $\tau=0.001 \mathrm{sec}$ & $\tau$ & $\tau=1 \mathrm{sec}$ & $\tau=0.1 \mathrm{sec}$ \\
\hline$\omega_{1}$ & $1.0 \mathrm{e}+013 *$ & $1.0 \mathrm{e}+012 *$ & $\omega_{1}$ & $1.0 \mathrm{e}+015 *$ & $1.0 \mathrm{e}+014 *$ \\
\hline$\omega_{2}$ & -1.1080 & -1.1080 & $\omega_{2}$ & -1.1080 & -1.1080 \\
\hline$\omega_{3}$ & 1.1080 & 1.1080 & $\omega_{3}$ & 1.1080 & 1.1080 \\
\hline$\omega_{4}$ & $0.0000+0.0000 i$ & $-0.0000+0.0000 i$ & $\omega_{4}$ & $-0.0000+0.0000 i$ & $0.0000+0.0000 i$ \\
\hline$\omega_{5}$ & $0.0000-0.0000 \mathrm{i}$ & $-0.0000-0.0000 i$ & $\omega_{5}$ & $-0.0000-0.0000 i$ & $0.0000-0.0000 i$ \\
\hline \multicolumn{3}{|c|}{$\begin{array}{l}\text { Table 3c. Cylindrical RF network antennas system roots } \\
\qquad \omega_{i}(\tau)\end{array}$} & \multicolumn{3}{|c|}{$\begin{array}{l}\text { Table } 3 d \text {. Cylindrical RF network antennas system } \\
\text { roots } \omega_{i}(\tau)\end{array}$} \\
\hline$\tau$ & $\tau=3 \mathrm{sec}$ & $\tau=2 \mathrm{sec}$ & $\tau$ & $\tau=5 \mathrm{sec}$ & $\tau=4 \mathrm{sec}$ \\
\hline$\omega_{1}$ & $1.0 \mathrm{e}+015^{*}$ & $1.0 \mathrm{e}+015^{*}$ & $\omega_{1}$ & $1.0 \mathrm{e}+015 *$ & $1.0 \mathrm{e}+015^{*}$ \\
\hline$\omega_{2}$ & 3.3240 & -2.2160 & $\omega_{2}$ & -5.5399 & 4.4319 \\
\hline$\omega_{3}$ & -3.3240 & 2.2160 & $\omega_{3}$ & 5.5399 & -4.4319 \\
\hline$\omega_{4}$ & $0+0.0000 i$ & $-0.0000+0.0000 i$ & $\omega_{4}$ & $0.0000+0.0000 i$ & $0+0.0000 i$ \\
\hline$\omega_{5}$ & $0-0.0000 \mathrm{i}$ & $-0.0000-0.0000 i$ & $\omega_{5}$ & $0.0000-0.0000 i$ & $0-0.0000 \mathrm{i}$ \\
\hline \multicolumn{3}{|c|}{$\begin{array}{l}\text { Table 3e. Cylindrical RF network antennas systen } \\
\text { roots } \omega_{i}(\tau)\end{array}$} & \multicolumn{3}{|c|}{$\begin{array}{l}\text { Table 3f. Cylindrical RF network antennas system roots } \\
\qquad \omega_{i}(\tau)\end{array}$} \\
\hline$\tau$ & $\tau=7 \mathrm{sec}$ & $\tau=6 \mathrm{sec}$ & $\tau$ & $\tau=9 \mathrm{sec}$ & $\tau=8 \mathrm{sec}$ \\
\hline$\omega_{1}$ & $1.0 \mathrm{e}+015^{*}$ & $1.0 \mathrm{e}+015^{*}$ & $\omega_{1}$ & $1.0 \mathrm{e}+015^{*}$ & $1.0 \mathrm{e}+015^{*}$ \\
\hline$\omega_{2}$ & -7.7559 & 6.6479 & $\omega_{2}$ & 9.9719 & 8.8639 \\
\hline$\omega_{3}$ & 7.7559 & -6.6479 & $\omega_{3}$ & -9.9719 & -8.8639 \\
\hline$\omega_{4}$ & $0.0000+0.0000 \mathrm{i}$ & $0+0.0000 i$ & $\omega_{4}$ & $0+0.0000 i$ & $0+0.0000 i$ \\
\hline$\omega_{5}$ & $0.0000-0.0000 i$ & $0-0.0000 i$ & $\omega_{5}$ & $0-0.0000 i$ & $0-0.0000 i$ \\
\hline
\end{tabular}

Table 3g. Cylindrical RF network antennas system roots $\omega_{i}(\tau)$

\begin{tabular}{|c|c|c|}
\hline$\tau$ & $\tau=0 \mathrm{sec}$ & $\tau=10 \mathrm{sec}$ \\
\hline$\omega_{1}$ & $1.0 \mathrm{e}+007^{*}$ & $1.0 \mathrm{e}+016^{*}$ \\
\hline$\omega_{2}$ & -3.3286 & -1.1080 \\
\hline$\omega_{3}$ & $-0.0000+3.3286 \mathrm{i}$ & 1.1080 \\
\hline$\omega_{4}$ & $-0.0000-3.3286 \mathrm{i}$ & $-0.0000+0.0000 \mathrm{i}$ \\
\hline$\omega_{5}$ & 3.3286 & $-0.0000-0.0000 \mathrm{i}$ \\
\hline
\end{tabular}

We can summery our $\omega_{i}(\tau)$ results for $\omega_{i}(\tau)>0$ and real number (ignore complex, negative, and imaginary values). We exclude from our table the high and real $\omega_{i}(\tau)$ values $\left(1.0 \mathrm{e}+007^{*}, 1.0 \mathrm{e}+012\right.$ $*, \ldots, 1.0 \mathrm{e}+016 *$ ) and add results for $\tau=15 \mathrm{sec}$ and $\tau=20 \mathrm{sec}$. 
Table 4. Cylindrical RF network antennas system Positive and real roots $\omega_{i}(\tau)$ values and $\sin (\omega \cdot \tau), \cos (\omega \cdot \tau)$ values

\begin{tabular}{|c|c|c|c|}
\hline$\tau[\mathrm{sec}]$ & $\omega$ & $\begin{array}{c}\sin (\omega \cdot \tau) \\
=\frac{-\omega^{3} \cdot \tau \cdot L \cdot C}{\left(\omega^{2} \cdot \tau^{2}+1\right)}\end{array}$ & $\begin{array}{c}\cos (\omega \cdot \tau) \\
=\frac{\omega^{2} \cdot L \cdot C}{\left(\omega^{2} \cdot \tau^{2}+1\right)}\end{array}$ \\
\hline 0 & 3.3286 & $0=0$ & $1 \neq 9.9 \mathrm{e}-15$ \\
\hline $0.001 . .1$ & 1.1080 & $\begin{array}{c}-1.22 \mathrm{e}-18 \ldots \\
-5.51 \mathrm{e}-16\end{array}$ & $\begin{array}{c}1.108 \mathrm{e}-15 \ldots \\
4.973 \mathrm{e}-16\end{array}$ \\
\hline 2 & 2.2160 & $-9.5 \mathrm{e}-16$ & $2.1 \mathrm{e}-16$ \\
\hline 3 & 3.3240 & $-9.9 \mathrm{e}-16$ & $9.9 \mathrm{e}-17$ \\
\hline 4 & 4.4319 & $-9.9 \mathrm{e}-16$ & $5.62 \mathrm{e}-17$ \\
\hline 5 & 5.5399 & $-9.9 \mathrm{e}-16$ & $3.6 \mathrm{e}-17$ \\
\hline 6 & 6.6479 & $-9.99-16$ & $2.5055 \mathrm{e}-17$ \\
\hline 7 & 7.7559 & $-9.9966 \mathrm{e}-16$ & $1.8413 \mathrm{e}-17$ \\
\hline 8 & 8.8639 & $-9.9980 \mathrm{e}-16$ & $1.4099 \mathrm{e}-17$ \\
\hline 9 & 9.9719 & $-9.9988 \mathrm{e}-16$ & $1.1141 \mathrm{e}-17$ \\
\hline 10 & 1.1080 & $-9.9193 \mathrm{e}-17$ & $8.9525 \mathrm{e}-18$ \\
\hline 15 & 1.6620 & $-9.9841 \mathrm{e}-17$ & $4.0048 \mathrm{e}-18$ \\
\hline 20 & 2.2160 & $-9.9950 \mathrm{e}-17$ & $2.2552 \mathrm{e}-18$ \\
\hline
\end{tabular}

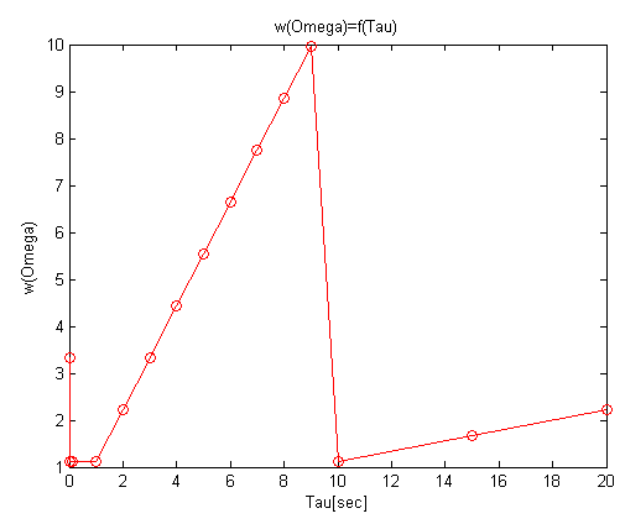

Figure5. Cylindrical RF network $F(\omega, \tau)$ function for $\tau_{1}=\tau_{2}=\tau$

Matlab: plot([0 0.001 0.010.11234567891015 20],[3.32861.10801.10801.10801.10802.2160 $3.32404 .43195 .53996 .64797 .75598 .86399 .97191 .10801 .66202 .2160]$,'-or'). We plot 3D function $F(\omega, \tau)=0 . \tau: 0 \rightarrow 10 ; \omega: 0 \rightarrow 100$. We define additional MATLAB script parameters $\omega \rightarrow \mathrm{w}, \tau \rightarrow \mathrm{t}$. 


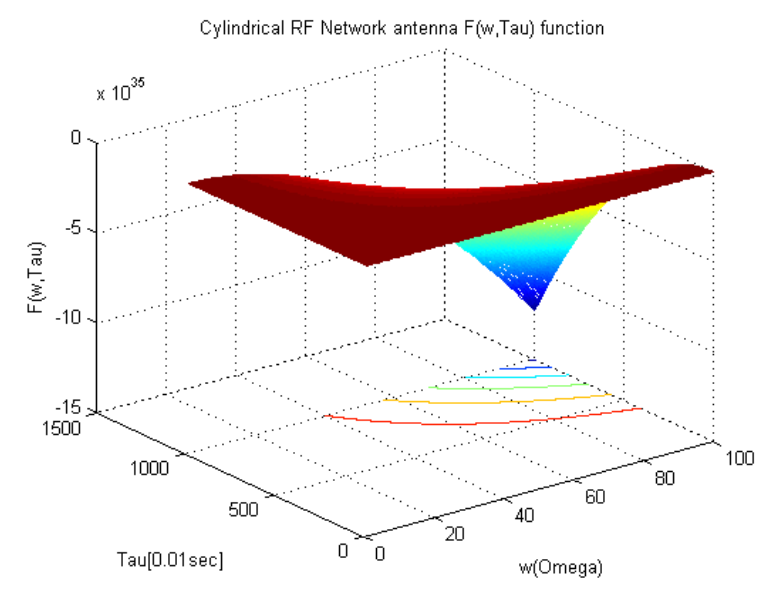

Figure 6. Cylindrical RF network $F(\omega, \tau)$ function for $\tau_{1}=\tau_{2}=\tau$

Matlab:[w,t]=meshgrid(1:1:100,0:0.01:10);C=2.47*1e-9;L=365.4*1e-9;f=w. ${ }^{*} w .{ }^{*} w .{ }^{*} w-$ w. ${ }^{*}{ }^{*}(\mathrm{t} . * \mathrm{t}) /(\mathrm{C} * \mathrm{~L} * \mathrm{C} * \mathrm{~L})-1 /\left(\mathrm{C} * \mathrm{~L}^{*} \mathrm{C} * \mathrm{~L}\right) ; \operatorname{meshc}(\mathrm{f}) ; \% \omega \rightarrow w, \tau \rightarrow t$

We get two possible real values for $\omega$ which fulfil $F(\omega, \tau)=0$

$F(\omega=3.3286$ or $\omega=1.1080 \ldots . .$. or $\omega=2.2160, \tau)=0 ; \tau \in[0.001 . .10]$ Next is to find those $\omega, \tau$ values which fulfil $\sin \theta(\tau)=\ldots$

$$
\begin{aligned}
& \sin (\omega \cdot \tau)=\frac{-P_{R} \cdot Q_{I}+P_{I} \cdot Q_{R}}{|Q|^{2}} \text { And } \cos \theta(\tau)=\ldots \\
& \cos (\omega \cdot \tau)=-\frac{\left(P_{R} \cdot Q_{R}+P_{I} \cdot Q_{I}\right)}{|Q|^{2}} ;|Q|^{2}=Q_{R}^{2}+Q_{I}^{2} \cdot \\
& \sin (\omega \cdot \tau)=\frac{-\omega^{3} \cdot \tau \cdot L \cdot C}{\left(\omega^{2} \cdot \tau^{2}+1\right)} ; \cos (\omega \cdot \tau)=\frac{\omega^{2} \cdot L \cdot C}{\left(\omega^{2} \cdot \tau^{2}+1\right)}
\end{aligned}
$$

$\frac{-\omega^{3} \cdot \tau \cdot L \cdot C}{\left(\omega^{2} \cdot \tau^{2}+1\right)}<0 \& \frac{\omega^{2} \cdot L \cdot C}{\left(\omega^{2} \cdot \tau^{2}+1\right)}>0$ then $\sin (\omega \cdot \tau)<0$ and $\cos (\omega \cdot \tau)>0 ; 2 \cdot \pi>\omega \cdot \tau>\frac{\pi}{2} \cdot 3$. We plot the stability switch diagram based on different delay values of our Cylindrical RF network antennas system.

$$
\begin{aligned}
\wedge^{-1}(\tau) & =\left(\frac{\partial \operatorname{Re} \lambda}{\partial \tau}\right)_{\lambda=i \cdot \omega}=\operatorname{Re}\left\{\frac{-2 \cdot\left[U+\tau \cdot|P|^{2}\right]+i \cdot F_{\omega}}{F_{\tau}+i \cdot 2 \cdot\left[V+\omega \cdot|P|^{2}\right]}\right\} \\
\wedge^{-1}(\tau) & =\left(\frac{\partial \operatorname{Re} \lambda}{\partial \tau}\right)_{\lambda=i \cdot \omega}=\frac{2 \cdot\left\{F_{\omega} \cdot\left(V+\omega \cdot P^{2}\right)-F_{\tau} \cdot\left(U+\tau \cdot P^{2}\right)\right\}}{F_{\tau}^{2}+4 \cdot\left(V+\omega \cdot P^{2}\right)^{2}}
\end{aligned}
$$

$\operatorname{sign}\left\{\wedge^{-1}(\tau)\right\}=\operatorname{sign}\left[\psi_{1}\right] \cdot \operatorname{sign}\left[\psi_{2}+\omega+\psi_{3}\right]$. We define the following new functions:

$$
\begin{array}{r}
g_{1}=\psi_{1} ; g_{2}=\psi_{2}+\omega+\psi_{3} \\
\operatorname{sign}\left\{\wedge^{-1}(\tau)\right\}=\operatorname{sign}\left[g_{1}\right] \cdot \operatorname{sign}\left[g_{2}\right]
\end{array}
$$




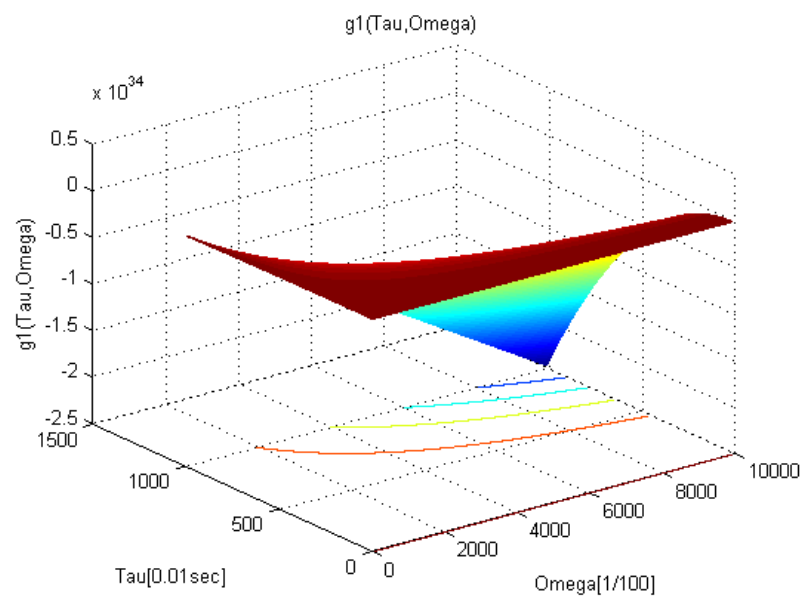

Figure 7. Cylindrical RF network $g_{1}(\tau, \omega)$ function for $\tau_{1}=\tau_{2}=\tau$.

Matlab: $[w, t]=$ meshgrid(1:.01:100,0:0.01:10);C=2.47*1e-9; L=365.4*1e-9;f=2*w.* $(2 * w . * w-$ (t.*t./(C*L*C*L)));meshc(f)

$\% \omega \rightarrow w, \tau \rightarrow t$

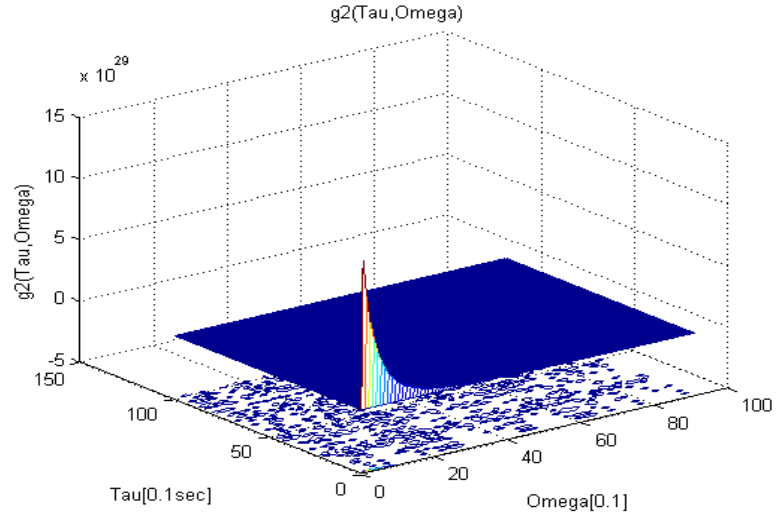

Figure 8. Cylindrical RF network $g_{2}(\tau, \omega)$ function for $\tau_{1}=\tau_{2}=\tau$.

Matlab: $[w, t]=m e s h g r i d(1: .1: 10,0: 0.1: 10) ; C=2.47 * 1 e-9 ; \mathrm{L}=365.4 * 1 \mathrm{e}-9 ; \mathrm{m}=\mathrm{w} .{ }^{*} \mathrm{t} . /\left(2 *{ }^{*} \cdot{ }^{*} \mathrm{w} .{ }^{*}\left(\mathrm{~L}^{*} \mathrm{C} \mathrm{C}^{*} \mathrm{~L}^{*} \mathrm{C}\right)-\mathrm{t} .{ }^{*} \mathrm{t}\right)$; $\mathrm{f}=\mathrm{t} .{ }^{*} \mathrm{~m}+\mathrm{w}+\left(\mathrm{m} .{ }^{*} \mathrm{t} . /\left(\mathrm{L}^{*} \mathrm{C} * \mathrm{~L} * \mathrm{C}\right)+\mathrm{w} . /\left(\mathrm{L}^{*} \mathrm{C} * \mathrm{~L}^{*} \mathrm{C}\right)\right) . /\left(\mathrm{w} .{ }^{*} \mathrm{w} .{ }^{*} \mathrm{w} .{ }^{*} \mathrm{w}\right) ; \mathrm{meshc}(\mathrm{f}) \% \omega \rightarrow w, \tau \rightarrow t$

$$
g(\text { Tau })=g_{1}(\text { Tau }) \cdot g_{2}(\text { Tau })=\wedge^{-1}(\tau)=\left(\frac{\partial \operatorname{Re} \lambda}{\partial \tau}\right)_{\lambda=i \cdot \omega}
$$

The stability switch occur only on those delay values $(\tau)$ which fit the equation: $\tau=\frac{\theta_{+}(\tau)}{\omega_{+}(\tau)}$ and $\theta_{+}(\tau)$ is the solution

$$
\text { Of } \sin \theta(\tau)=\frac{-\omega^{3} \cdot \tau \cdot L \cdot C}{\left(\omega^{2} \cdot \tau^{2}+1\right)} ; \cos \theta(\tau)=\frac{\omega^{2} \cdot L \cdot C}{\left(\omega^{2} \cdot \tau^{2}+1\right)}
$$


When $\omega=\omega_{+}(\tau)$ if only $\omega_{+}$is feasible. Additionally When all Cylindrical RF network antennas parameters are known and the stability switch due to various time delay values $\tau$ is describe in the below expression (Appendix E):

$$
\begin{aligned}
& \operatorname{sign}\left\{\wedge^{-1}(\tau)\right\}=\operatorname{sign}\left\{F_{\omega}(\omega(\tau), \tau)\right\} \cdot \operatorname{sign}\left\{\tau \cdot \omega_{\tau}(\omega(\tau))\right. \\
& \left.+\omega(\tau)+\frac{U(\omega(\tau)) \cdot \omega_{\tau}(\omega(\tau))+V(\omega(\tau))}{|P(\omega(\tau))|^{2}}\right\}
\end{aligned}
$$

Remark: we know $F(\omega, \tau)=0$ implies it roots $\omega_{i}(\tau)$ and finding those delays values $\tau$ which $\omega_{i}$ is feasible. There are $\tau$ values which $\omega_{i}$ is complex or imaginary number, then unable to analyse stability [4] [5].

\section{Discussion}

In this paper, we consider Cylindrical RF network antennas system. Due to RF antenna copper leg parasitic effect we get copper leg's current and current derivative with delay $\tau 1-k$ and $\tau 2-k$ ( $k$ is leg number index, $k=1, \ldots, 16)$.. Those delays causes to stability switching for our Cylindrical RF network antennas. We draw our Cylindrical RF network antennas equivalent circuit and get system differential equations. Our variables are $\mathrm{Y}, \mathrm{X}$ which are function of RF antenna copper leg's current and current derivative. Our system dynamic behavior is dependent on circuit overall parameters and parasitic delay in time. We keep all circuit parameters fix and change, parasitic delay over various values $\tau \in[0.001 . .10]$. Our analysis results extend that of in the way that it deals with stability switching for different delay values. This implies that our system behavior of the circuit cannot inspect by short analysis and we must study the full system. Several very important issues and possibilities were left out of the present paper. One possibility is the stability switching by circuit parameters. Every circuit's parameter variation can change our system dynamic and stability behavior. This case can be solved by the same methods combined with alternative and more technical hypotheses. Moreover, numerical simulations for the Cylindrical RF network antennas model studied in references suggest that this result can be extended to enhance models with more general functions. Still another extension of our results would be to treat the case of delayed Cylindrical RF network antennas leg's higher derivative degree of current. It would be extremely desirable to confirm these cases by mathematical proofs.

\section{Conclusion}

Cylindrical RF network antennas system is characterized by parasitic effects which can influence Cylindrical RF network antennas system stability in time. There are two main Cylindrical RF network antennas variables which are affected by antenna legs parasitic effects, $Y$ and $X$ functions of antenna leg's currents and currents derivatives respectively. Each Cylindrical RF network antennas system variable under parasitic effects is characterized by time delay respectively. The two time delays are not the same, but can be categorized to some sub cases due to antenna leg parasitic behavior.

The first case we analyze is when there is delay in Cylindrical RF network antennas leg's current and no delay in antennas leg's current derivative or opposite. The second case we analyze is when there is delay both in Cylindrical RF network antennas leg's current and current time derivative [4] [5]. For simplicity of our analysis we consider in the second case all delays are the same (there is a difference but it is 
neglected in our analysis). In each case we derive the related characteristic equation. The characteristic equation is dependent on Cylindrical RF network antennas system overall parameters and parasitic time delay. Upon mathematics manipulation and [BK] theorems and definitions we derive the expression which gives us a clear picture on Cylindrical RF network antennas map. The stability map gives all possible options for stability segments, each segment belongs to different time delay value segment. Cylindrical RF network antennas system's stability analysis can be influenced either by system overall parameter values. We left this analysis and do not discuss it in the current article.

\section{Appendix A : Lemma 1.1}

Assume that $\omega(\tau)$ is a positive and real root of $F(\omega, \tau)=0$

Defined for $\tau \in I$, which is continuous and differentiable. Assume further that if $\lambda=i \cdot \omega, \omega \in R$, then $P_{n}(i \cdot \omega, \tau)+Q_{n}(i \cdot \omega, \tau) \neq 0, \tau \in \mathrm{R}$ hold true. Then the functions $S_{n}(\tau), n \in N_{0}$, are continuous and differentiable on I.

\section{Appendix B : Theorem 1.2}

Assume that $\omega(\tau)$ is a positive real root of $F(\omega, \tau)=0$ defined for $\tau \in I, I \subseteq R_{+0}$, and at some $\tau^{*} \in I$ , $S_{n}\left(\tau^{*}\right)=0$

For some $n \in N_{0}$ then a pair of simple conjugate pure imaginary roots $\lambda_{+}\left(\tau^{*}\right)=i \cdot \omega\left(\tau^{*}\right), \lambda_{-}\left(\tau^{*}\right)=-i \cdot \omega\left(\tau^{*}\right)$ of $D(\lambda, \tau)=0$ exist at $\tau=\tau^{*}$ which crosses the imaginary axis from left to right if $\delta\left(\tau^{*}\right)>0$ and cross the imaginary axis from right to left if $\delta\left(\tau^{*}\right)<0$ where

$$
\begin{aligned}
& \delta\left(\tau^{*}\right)=\operatorname{sign}\left\{\left.\frac{d \operatorname{Re} \lambda}{d \tau}\right|_{\lambda=i \omega\left(\tau^{*}\right)}\right\}= \\
& \operatorname{sign}\left\{F_{\omega}\left(\omega\left(\tau^{*}\right), \tau^{*}\right)\right\} \cdot \operatorname{sign}\left\{\left.\frac{d S_{n}(\tau)}{d \tau}\right|_{\tau=\tau^{*}}\right\}
\end{aligned}
$$

The theorem becomes

$$
\operatorname{sign}\left\{\left.\frac{d \operatorname{Re} \lambda}{d \tau}\right|_{\lambda=i \omega \pm}\right\}=\operatorname{sign}\left\{ \pm \Delta^{1 / 2}\right\} \cdot \operatorname{sign}\left\{\left.\frac{d S_{n}(\tau)}{d \tau}\right|_{\tau=\tau^{*}}\right\}
$$

\section{Appendix C : Theorem 1.3}

The characteristic equation: $\tau_{1}=\tau, \tau_{2}=0 ; \tau_{1}=0, \tau_{2}=\tau$

$$
\begin{aligned}
& D(\lambda, \tau)=\lambda^{2}+a(\tau) \cdot \lambda+b(\tau) \cdot \lambda \cdot e^{-\lambda \cdot \tau}+c(\tau)+d(\tau) \cdot e^{-\lambda \cdot \tau} \\
& D\left(\lambda, \tau_{1}, \tau_{2}\right)=\lambda^{2}+\lambda \cdot \frac{1}{C 1 \cdot R 1} \cdot e^{-\lambda \cdot \tau_{2}}+\frac{1}{C 1 \cdot f_{\#}} \cdot e^{-\lambda \cdot\left(\tau_{1}+\tau_{2}\right)}
\end{aligned}
$$

Has a pair of simple and conjugate pure imaginary roots 
$\lambda= \pm \omega\left(\tau^{*}\right), \omega\left(\tau^{*}\right)$ real at $\tau^{*} \in I$ if $S_{n}\left(\tau^{*}\right)=\tau^{*}-\tau_{n}\left(\tau^{*}\right)=0$ for some $n \in N_{0}$. If $\omega\left(\tau^{*}\right)=\omega_{+}\left(\tau^{*}\right)$, this pair of simple conjugate pure imaginary roots crosses the imaginary axis from left to right if $\delta_{+}\left(\tau^{*}\right)>0$ and crosses the imaginary axis from right to left if $\delta_{+}\left(\tau^{*}\right)<0$ where

$$
\delta_{+}\left(\tau^{*}\right)=\operatorname{sign}\left\{\left.\frac{d \operatorname{Re} \lambda}{d \tau}\right|_{\lambda=i \omega_{+}\left(\tau^{*}\right)}\right\}=\operatorname{sign}\left\{\left.\frac{d S_{n}(\tau)}{d \tau}\right|_{\tau=\tau^{*}}\right\}
$$

If $\omega\left(\tau^{*}\right)=\omega_{-}\left(\tau^{*}\right)$, this pair of simple conjugate pure imaginary roots cross the imaginary axis from left to right if

$\delta_{-}\left(\tau^{*}\right)>0$ and crosses the imaginary axis from right to left

If $\delta_{-}\left(\tau^{*}\right)<0$ where

$$
\delta_{-}\left(\tau^{*}\right)=\operatorname{sign}\left\{\left.\frac{d \operatorname{Re} \lambda}{d \tau}\right|_{\lambda=i \omega_{-}\left(\tau^{*}\right)}\right\}=-\operatorname{sign}\left\{\left.\frac{d S_{n}(\tau)}{d \tau}\right|_{\tau=\tau^{*}}\right\}
$$

If $\omega_{+}\left(\tau^{*}\right)=\omega_{-}\left(\tau^{*}\right)=\omega\left(\tau^{*}\right)$ then $\Delta\left(\tau^{*}\right)=0$ and $\operatorname{sign}\left\{\left.\frac{d \operatorname{Re} \lambda}{d \tau}\right|_{\lambda=i \omega\left(\tau^{*}\right)}\right\}=0$, the same is true when $S_{n}^{\prime}\left(\tau^{*}\right)=0$

The following result can be useful in identifying values of $\tau$

Where stability switches happened.

Appendix D : Theorem 1.4

Assume that for all $\tau \in I, \omega(\tau)$ is defined as a solution of

$$
\begin{aligned}
& F(\omega, \tau)=0 \text { then } \delta_{ \pm}(\tau)=\operatorname{sign}\{\left. \pm \Delta^{1 / 2}(\tau)\right\} \cdot \operatorname{sign} D_{ \pm}(\tau) \\
& D_{ \pm}(\tau)=\omega_{ \pm}^{2} \cdot\left[\left(\omega_{ \pm}^{2} \cdot b^{2}+d^{2}\right)+a^{\prime} \cdot\left(c-\omega_{ \pm}^{2}\right)+b \cdot d^{\prime}-b^{\prime} \cdot d-a \cdot c^{\prime}\right] \\
&+\omega_{ \pm} \cdot \omega_{ \pm}^{\prime} \cdot\left[\tau \cdot\left(\omega_{ \pm}^{2} \cdot b^{2}+d^{2}\right)-b \cdot d+a \cdot\left(c-\omega_{ \pm}^{2}\right)+2 \cdot \omega_{ \pm}^{2} \cdot a\right] \\
& a^{\prime}=\frac{d a(\tau)}{d \tau} ; \mathrm{b}^{\prime}=\frac{d b(\tau)}{d \tau} ; \mathrm{c}^{\prime}=\frac{d c(\tau)}{d \tau} ; \mathrm{d}^{\prime}=\frac{d d(\tau)}{d \tau}
\end{aligned}
$$

Appendix E: We need to approve the following expression

$$
\begin{gathered}
\operatorname{sign}\left\{\wedge^{-1}(\tau)\right\}=\operatorname{sign}\left\{F_{\omega}(\omega(\tau), \tau)\right\} \cdot \operatorname{sign}\left\{\tau \cdot \omega_{\tau}(\omega(\tau))\right. \\
\left.+\omega(\tau)+\frac{U(\omega(\tau)) \cdot \omega_{\tau}(\omega(\tau))+V(\omega(\tau))}{|P(\omega(\tau))|^{2}}\right\} \\
\text { The basic assumption: } \wedge^{-1}(\tau)=\left(\frac{\partial \operatorname{Re} \lambda}{\partial \tau}\right)_{\lambda=i \cdot \omega} \\
\wedge^{-1}(\tau)=\left(\frac{\partial \operatorname{Re} \lambda}{\partial \tau}\right)_{\lambda=i \cdot \omega}=\frac{2 \cdot\left\{F_{\omega} \cdot\left(V+\omega \cdot P^{2}\right)-F_{\tau} \cdot\left(U+\tau \cdot P^{2}\right)\right\}}{F_{\tau}^{2}+4 \cdot\left(V+\omega \cdot P^{2}\right)^{2}}
\end{gathered}
$$




$$
\begin{aligned}
& \operatorname{sign}\left\{F_{\tau}^{2}+4 \cdot\left(V+\omega \cdot P^{2}\right)^{2}\right\}>0 \text { and } \omega_{\tau}=-\frac{F_{\tau}}{F_{\omega}} \text { then } \\
& \\
& \quad \operatorname{sign}\left\{\wedge^{-1}(\tau)\right\}=\operatorname{sign}\left\{\left(\frac{\partial \operatorname{Re} \lambda}{\partial \tau}\right)_{\lambda=i \cdot \omega}\right\} \\
&=\operatorname{sign}\left\{F_{\omega} \cdot\left(V+\omega \cdot P^{2}\right)-F_{\tau} \cdot\left(U+\tau \cdot P^{2}\right)\right\} \\
& \operatorname{sign}\left\{\wedge^{-1}(\tau)\right\}=\operatorname{sign}\left\{F_{\omega} \cdot\left\{\left(V+\omega \cdot P^{2}\right)-\frac{F_{\tau}}{F_{\omega}} \cdot\left(U+\tau \cdot P^{2}\right)\right\}\right\} \\
& \\
& \operatorname{sign}\left\{\wedge^{-1}(\tau)\right\}=\operatorname{sign}\left\{F_{\omega} \cdot\left\{\left(V+\omega \cdot P^{2}\right)+\omega_{\tau} \cdot\left(U+\tau \cdot P^{2}\right)\right\}\right\} \\
& \operatorname{sign}\left\{\wedge^{-1}(\tau)\right\}=\operatorname{sign}\left\{F_{\omega} \cdot\left\{V+\omega_{\tau} \cdot U+\omega \cdot P^{2}+\omega_{\tau} \cdot \tau \cdot P^{2}\right\}\right\} \\
& \operatorname{sign}\left\{\wedge^{-1}(\tau)\right\}=\operatorname{sign}\left\{P^{2} \cdot F_{\omega} \cdot\left\{\frac{V+\omega_{\tau} \cdot U}{P^{2}}+\omega+\omega_{\tau} \cdot \tau\right\}\right\} \\
& \operatorname{sign}\left\{\wedge^{-1}(\tau)\right\}=\operatorname{sign}\left\{P^{2}\right\} \cdot \operatorname{sign}\left\{F_{\omega}\right\} \\
& \cdot \operatorname{sign}\left\{\frac{V+\omega_{\tau} \cdot U}{P^{2}}+\omega+\omega_{\tau} \cdot \tau\right\} ; \operatorname{sign}\left\{P^{2}\right\}>0 \\
& \operatorname{sign}\left\{\wedge^{-1}(\tau)\right\}=\operatorname{sign}\left\{F_{\omega}\right\} \cdot \operatorname{sign}\left\{\frac{V+\omega_{\tau} \cdot U}{P^{2}}+\omega+\omega_{\tau} \cdot \tau\right\} \\
& \operatorname{REFERENCS}
\end{aligned}
$$

\section{REFERENCES}

[1]. Ch Hollenstein, Ph Guittienne and A A Howling, Resonant RF network antennas for large-area and largevolume inductively coupled plasma sources, Plasma Sources Sci. Technol. 22 (2013) 055021 (10pp).

[2]. Yuri A. Kuznetsov, Elelments of Applied Bifurcation Theory. Applied Mathematical Sciences. Springer 1995.

[3]. Jack K. Hale., Huseyin Kocak. Dynamics and Bifurcations.Texts in Applied Mathematics, Vol. 3. SpringerVerlag 1991.

[4]. Steven H. Strogatz, Nonlinear Dynamics and Chaos. Westview press. 1994

[5]. Kuang, Y., 1993. Delay Differential Equations with Applications in Population Dynamics. Academic Press, Boston.

[6]. Beretta E., Kuang, Y., 2002. Geometric stability switch criteria in delay differential systems with delay dependent parameters. SIAM J. Math. Anal. 33, 1144-1165.

[7]. Gerard looss., Daniel D.Joseph. Elementary stability and Bifurcation theory. Springer 1980.

[8]. John Guckenheimer., Philip Holmes. Nonlinear oscillations, dynamical systems, and bifurcation of vector fields. Applied Mathematical Sciences 42. Springer 1983.

[9]. Lawrence Perko. Differential equations and dynamics systems, Texts in applied mathematics 7. Springer 1991. 
Ofer Aluf; Cylindrical RF Network Antennas for Coupled Plasma Sources Copper Legs Delayed in Time System Stability Analysis, Transactions on Networks and Communications, Volume 2 No 5, Oct (2014); pp: 116-146

[10]. Kuang Jiaoxun and Cong Yuhao. Stability of numerical methods for delay differential equations, since press USA Inc, 2005. 